\title{
Theoretical status and prospects for top-quark pair production at hadron colliders
}

\author{
S. Moch ${ }^{1}$ and P. Uwer ${ }^{2}$ \\ ${ }^{1}$ Deutsches Elektronensynchrotron DESY, Platanenallee 6, D-15738 Zeuthen, Germany \\ ${ }^{2}$ Institut für Theoretische Teilchenphysik, Universität Karlsruhe, D-76128 Karlsruhe, Germany
}

(Received 27 May 2008; revised manuscript received 8 July 2008; published 6 August 2008)

\begin{abstract}
We present an update of the theoretical predictions for the cross section of top-quark pair production at Tevatron and LHC. In particular, we employ improvements due to soft-gluon resummation at next-tonext-to-leading logarithmic accuracy. We expand the resummed results and derive analytical finite-order cross sections through next-to-next-to-leading order which are exact in all logarithmically enhanced terms near threshold. These results are the best present estimates for the top-quark pair production cross section. We investigate the scale dependence as well as the sensitivity on the parton luminosities.
\end{abstract}

DOI: 10.1103/PhysRevD.78.034003

PACS numbers: $12.38 . \mathrm{Bx}, 13.85 . \mathrm{Ni}$

\section{INTRODUCTION}

Top-quark pair production at the LHC is important as the collider will accumulate very high statistics for this process. In the initial low luminosity run $(\sim 10 /$ (fb year) $)$ approximately $8 \times 10^{6}$ top-quark pairs will be produced per year $[1,2]$. This data will allow for numerous measurements, e.g. of the top-quark mass, the electric charge of the top-quark, or the weak couplings. Furthermore the data will allow precise tests of the production and the subsequent decay mechanism including anomalous couplings and top-quark spin correlations. See, for example, Ref. [3] for a recent review on top-quark physics at hadron colliders.

A necessary prerequisite which all these studies share in common is, of course, a detailed understanding of the production process. In quantum chromodynamics (QCD) this includes the radiative corrections to the cross section of heavy-quark hadroproduction at the next-to-leading order (NLO) [4-6] together with its scale dependence as well as the dependence on the parton luminosities through the parton distribution functions (PDFs) of the proton. Further improvements of the perturbative stability through resummation of large Sudakov logarithms to next-to-leading logarithmic (NLL) accuracy have been considered as well $[7,8]$ and employed to generate approximate results at the next-to-next-to-leading order (NNLO) in QCD [9]. Very recently also an estimate of bound state effects has been presented [10].

In particular, our knowledge on the parton luminosities has constantly improved over the past years. Thus, it is an imminent question how these improvements in the determination of the parton distribution functions from global fits affect predictions for physical cross sections at LHC, which are sensitive to the gluon distribution function in the regime where $x \approx 2.5 \times 10^{-2}$. For LHC observables this aspect has been quantitatively approached very recently by investigating correlations of rates for top-quark pair production with other cross sections [11]. For Tevatron, which to date has provided us with a lot of information on the topquark, most prominently a very precise determination [12] of its mass, $m_{t}=172.6 \pm 0.8$ (stat) \pm 1.1 (syst) GeV, the cross section $\sigma_{p p \rightarrow t \bar{t} X}$ in Eq. (1) had been studied some time ago $[13,14]$. However, due to changes in the available PDF sets from global fits, an update also seems to be in order here as well.

It is the aim of this article to review theoretical predictions for the production cross sections of top-quark pairs at Tevatron and LHC and to establish the present theoretical uncertainty. We provide an update of the NLL resummed cross section as defined in Ref. [8] (and also used in Ref. [14]). Subsequently, we extend these results to the next-to-next-to-leading logarithmic (NNLL) accuracy and derive approximate NNLO cross sections thereby improving previous calculations $[9,13]$. At two loops we are thus in a position to present all logarithmically enhanced terms near threshold and to assess their phenomenological impact by studying the quality of the perturbative expansion, i.e. the properties of apparent convergence and the stability under scale variations. This seems particularly interesting considering not only the anticipated experimental precision at LHC $[1,2]$ but also in view of recent activities aiming at complete NNLO QCD predictions for heavyquark hadroproduction [15-19].

The paper is organized as follows. In Sec. II we set the stage and study the threshold sensitivity of the inclusive hadronic cross section for top-quark pair production. Subsequently, we provide updates of Refs. $[8,14]$ employing recent sets of PDFs. In Sec. III we extend the resummed cross section to NNLL accuracy and calculate the complete logarithmic dependence of the cross section near threshold (including the Coulomb corrections). Together with the exact NNLO scale dependence of Ref. [9], these results are the best present estimates for the hadroproduction cross section of top-quark pairs. We conclude in Sec. IV and give some relevant formulas in Appendix A. In addition, cross section predictions using different approximations for individual PDFs are also listed in Appendix B. 


\section{THEORY STATUS}

Throughout this article, we restrict ourselves to the inclusive hadronic cross section $\sigma_{p p \rightarrow t \bar{t} X}$ (see e.g. Ref. [20] for recent work on top-quark pair invariant mass distributions). Denoting the hadronic center-ofmass energy squared by $s_{\text {had }}$ and the top-quark mass by $m_{t}$, the total hadronic cross section for top-quark pair production is obtained through

$$
\begin{aligned}
\sigma_{p p \rightarrow t \bar{t} X}\left(s_{\mathrm{had}}, m_{t}^{2}\right)= & \sum_{i, j=q, \bar{q}, g} \int_{4 m_{t}^{2}}^{s_{\text {had }}} d \hat{s} L_{i j}\left(\hat{s}, s_{\mathrm{had}}, \mu_{f}^{2}\right) \\
& \times \hat{\sigma}_{i j \rightarrow t \bar{t}}\left(\hat{s}, m_{t}^{2}, \mu_{f}^{2}, \mu_{r}^{2}\right) .
\end{aligned}
$$

The parton luminosities $L_{i j}\left(\hat{s}, s_{\text {had }}, \mu_{f}^{2}\right)$ are defined through

$$
L_{i j}\left(\hat{s}, s_{\text {had }}, \mu_{f}^{2}\right)=\frac{1}{s_{\text {had }}} \int_{\hat{s}}^{s_{\text {had }}} \frac{d s}{s} f_{i / p}\left(\mu_{f}^{2}, \frac{s}{s_{\text {had }}}\right) f_{j / p}\left(\mu_{f}^{2}, \frac{\hat{s}}{s}\right),
$$

where $f_{i / p}\left(x, \mu_{f}^{2}\right)$ is the PDF describing the density of partons of flavor $i$ in the proton $p$ carrying a fraction $x$ of the initial proton momentum, at factorization scale $\mu_{f}$. Note that we have included $s_{\text {had }}$ into the definition of $L_{i j}$ to allow an easy comparison of the luminosity function between different colliders, in particular, LHC and Tevatron. The sum in Eq. (1) runs over all massless parton flavors and the top-quark mass used is the so-called pole mass.

Within the context of perturbative QCD the standard way to estimate the theoretical uncertainty for the inclusive hadronic cross section $\sigma_{p p \rightarrow t \bar{t} X}$ in Eq. (1) is based on the residual dependence on the factorization/renormalization scale $\mu_{f} / \mu_{r}$. Starting from the available predictions to a certain order in perturbation theory, it is common practice to identify the factorization scale with the renormalization scale (i.e. $\mu_{f}=\mu_{r} \equiv \mu$ ) and to estimate the effect of uncalculated higher orders by varying $\mu$ in the interval $\left[m_{t} / 2,2 m_{t}\right]$. For a given global PDF fit in contrast, the uncertainties which stem from uncertainties of the experimental data used in the fits are treated systematically by a family of $n_{\mathrm{PDF}}$ pairs of PDFs, where $n_{\mathrm{PDF}}$ is the number of parameters used in the fit. Then, the systematic uncertainty for the observable $O$ under consideration is estimated by (e.g. $[11,21])$

$$
\Delta O=\frac{1}{2} \sqrt{\sum_{k=1, n_{\mathrm{PDF}}}\left(O_{k+}-O_{k-}\right)^{2}} .
$$

Here the observable $O_{k \pm}$ are obtained by using the parton distribution functions $f_{i / p}^{k \pm}$ obtained by a "statistical" $\pm 1 \sigma$ variation of the $k$ th fit parameter after diagonalization of the correlation matrix. (Strictly speaking, the fit parameters are varied by an amount which the authors of the corresponding PDF set take to be equivalent to a $\pm 1 \sigma$ variation.) To end up with an estimate of the overall uncertainty, the uncertainty coming from the PDFs has to be combined with the uncertainty due to uncalculated higher orders. Given that the two uncertainties are very different from each other, in one case we are faced with the traces of an experimental uncertainty-in the other case we have the systematic uncertainty due to missing higher order corrections which clearly do not follow any statistical law. Adding in quadrature the two uncertainties therefore seems to be inappropriate and we use a linear combination of the uncertainties as a conservative estimate for the total uncertainty of the top-quark pair cross section:

$$
\sigma\left(2 m_{t}\right)-\Delta \sigma_{\mathrm{PDF}}\left(2 m_{t}\right) \leq \sigma \leq \sigma\left(m_{t} / 2\right)+\Delta \sigma_{\mathrm{PDF}}\left(m_{t} / 2\right),
$$

where $\Delta \sigma_{\mathrm{PDF}}$ is computed according to Eq. (3). The NLO QCD corrections for the partonic cross sections $\hat{\sigma}_{i j \rightarrow t \bar{t}}$ in Eq. (1) (known for a long time [4-6]) provide the first instance in this procedure where a meaningful error can be defined through Eq. (4).

Thus, let us start our discussion of the various theoretical uncertainties by reviewing at NLO in QCD some basic aspects concerning the parton luminosities $L_{i j}$ as defined in Eq. (2) at LHC (Fig. 1) and Tevatron (Fig. 2). At LHC the highest flux is provided by the quark-gluon initial state (first plot of Fig. 1). However, as can be seen from the cross section plot of Fig. 1, the parton-level cross section $\hat{\sigma}_{q g \rightarrow t \bar{t}}$ is much smaller than for the $q \bar{q}$ - or $g g$-initiated processes, since the $q g$ channel is of order $\alpha_{s}^{3}$ and thus formally a NLO correction. As a consequence, in the total hadronic cross section the $q g$ channel gives only a contribution at the percent level (see the second last plot in Fig. 1). In principle, the same argument applies for the $\bar{q} g$ channel, however its contribution to the hadronic cross section is even further suppressed because of the smaller parton luminosity.

The second largest parton flux is delivered by the $g g$ channel. In combination with the large partonic cross section $\hat{\sigma}_{g g \rightarrow t \bar{t}}$ this is the most important channel at LHC, resulting in about $90 \%$ of all top-quark pairs produced via gluon fusion. Close to threshold the uncertainty of the gluon flux [estimated using Eq. (3), see also discussion there] is about $3 \%$ and at $1 \mathrm{TeV}$ it grows to almost $10 \%$. However, given that the $g g$ channel is largely saturated at parton energies $\sqrt{\hat{s}} \simeq 1 \mathrm{TeV}$, the large PDF uncertainty above $1 \mathrm{TeV}$ does not have a significant impact on the overall uncertainty of $\sigma_{p p \rightarrow t \bar{X} X}$ in Eq. (1) (see the last plot in Fig. 1).

The parton luminosity $L_{q \bar{q}}$ ranks third at LHC. Close to threshold the $q \bar{q}$ flux is suppressed by roughly a factor 10 compared to $L_{g g}$. The corresponding parton cross section $\hat{\sigma}_{q \bar{q} \rightarrow t \bar{t}}$ vanishes in the high energy limit in contrast to the $g g$ and $q g$ case where the partonic cross sections approach a constant at high energies. Thus, the $q \bar{q}$ contribution to the hadronic cross section saturates well below $1 \mathrm{TeV}$ and adds the known $10 \%$ at LHC. Because of the small numerical 
contribution of the $q \bar{q}$ and $q g$ channels, the PDF uncertainty of the hadronic cross section $\sigma_{p p \rightarrow t \bar{t} X}$ is entirely dominated by the uncertainty of $L_{g g}$.

At Tevatron, the situation is reversed (see Fig. 2). The luminosities $L_{i j}$ are ordered in magnitude according to $L_{q \bar{q}}>L_{q g}>L_{g g}$. This makes the $q \bar{q}$ channel by far the dominant one contributing $85 \%$ to the hadronic cross
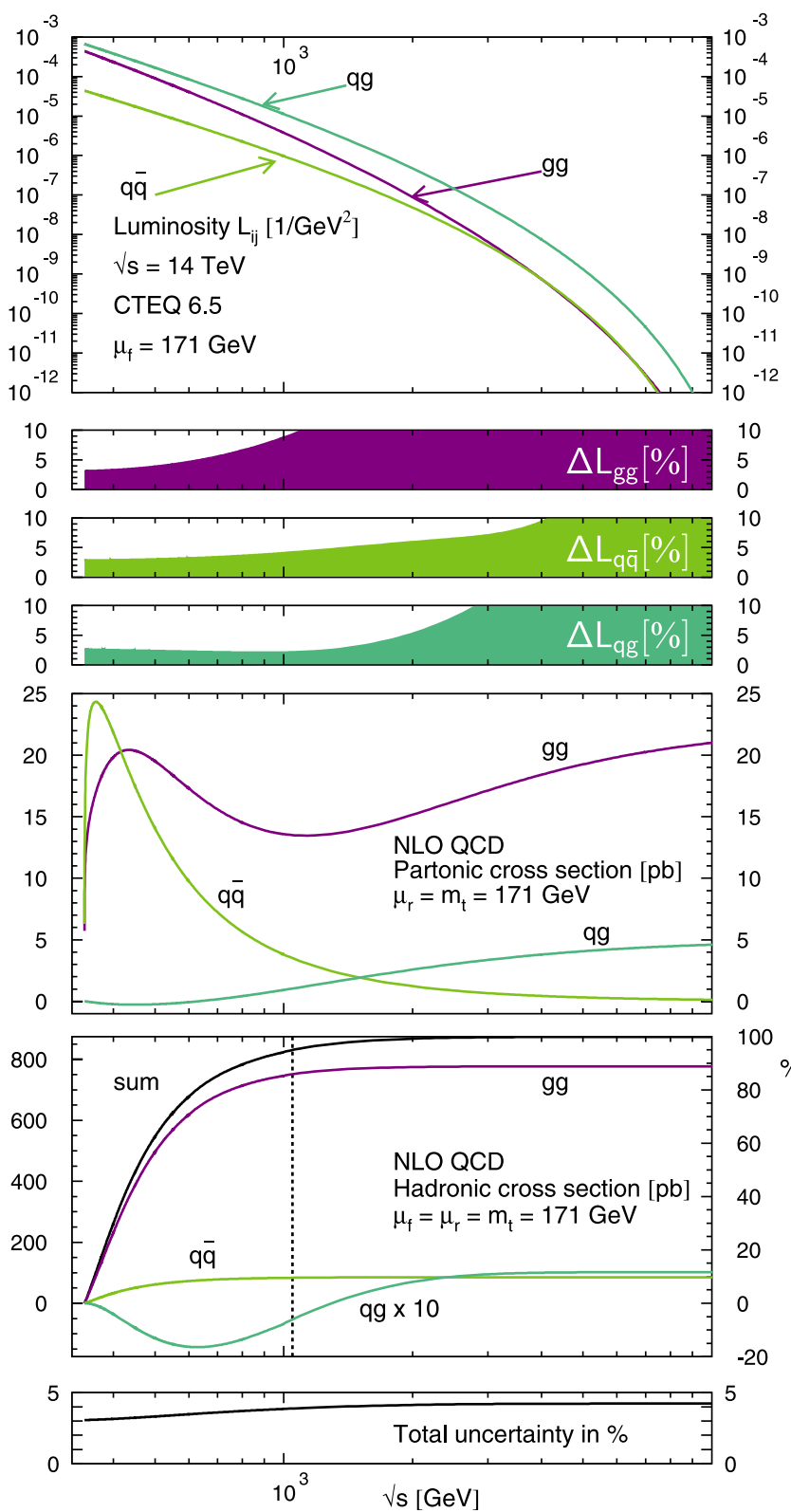

FIG. 1 (color online). The parton luminosity $L_{i j}$ with the individual PDF uncertainties (upper plots) and the parton cross sections $\hat{\sigma}_{i j \rightarrow t \bar{t}}$ at NLO in QCD (third plot from below) as a function of the parton energy $\sqrt{s}$. The lower plots scan the total cross section $\sigma\left(s_{\text {had }}, m_{t}^{2} ; s_{\max }\right)$ (the total PDF uncertainty) as a function of $\sqrt{s_{\max }}$ for LHC. We use $\sqrt{s_{\mathrm{had}}}=14 \mathrm{TeV}, m_{t}=$ $171 \mathrm{GeV}, \mu=m_{t}$, and the CTEQ6.5 PDF set. The dashed line indicates the value of $\sqrt{s_{\max }}$ for which the cross section is saturated to $95 \%$ section $\sigma_{p p \rightarrow t \bar{t} X}$, while gluon fusion almost makes up for the rest. Although the PDF uncertainty of the $q \bar{q}$ flux is only $3 \%-4 \%$ at low energies the overall PDF uncertainty of the top-quark cross section $\sigma_{p p \rightarrow t \bar{t} X}$ is large, because of the sensitivity to the gluon PDF content at large- $x$, which is still poorly constrained at present (see Fig. 2).

Finally, it is interesting to determine the value of $s_{\max }$ for which the cross section

$$
\begin{aligned}
\sigma\left(s_{\text {had }}, m_{t}^{2} ; s_{\text {max }}\right)= & \sum_{i, j=q, \bar{q}, g} \int_{4 m_{t}^{2}}^{s_{\max }} d \hat{s} L_{i j}\left(\hat{s}, s_{\text {had }}, \mu_{f}^{2}\right) \\
& \times \hat{\sigma}_{i j \rightarrow t \bar{t}}\left(\hat{s}, m_{t}^{2}, \mu_{f}^{2}, \mu_{r}^{2}\right)
\end{aligned}
$$

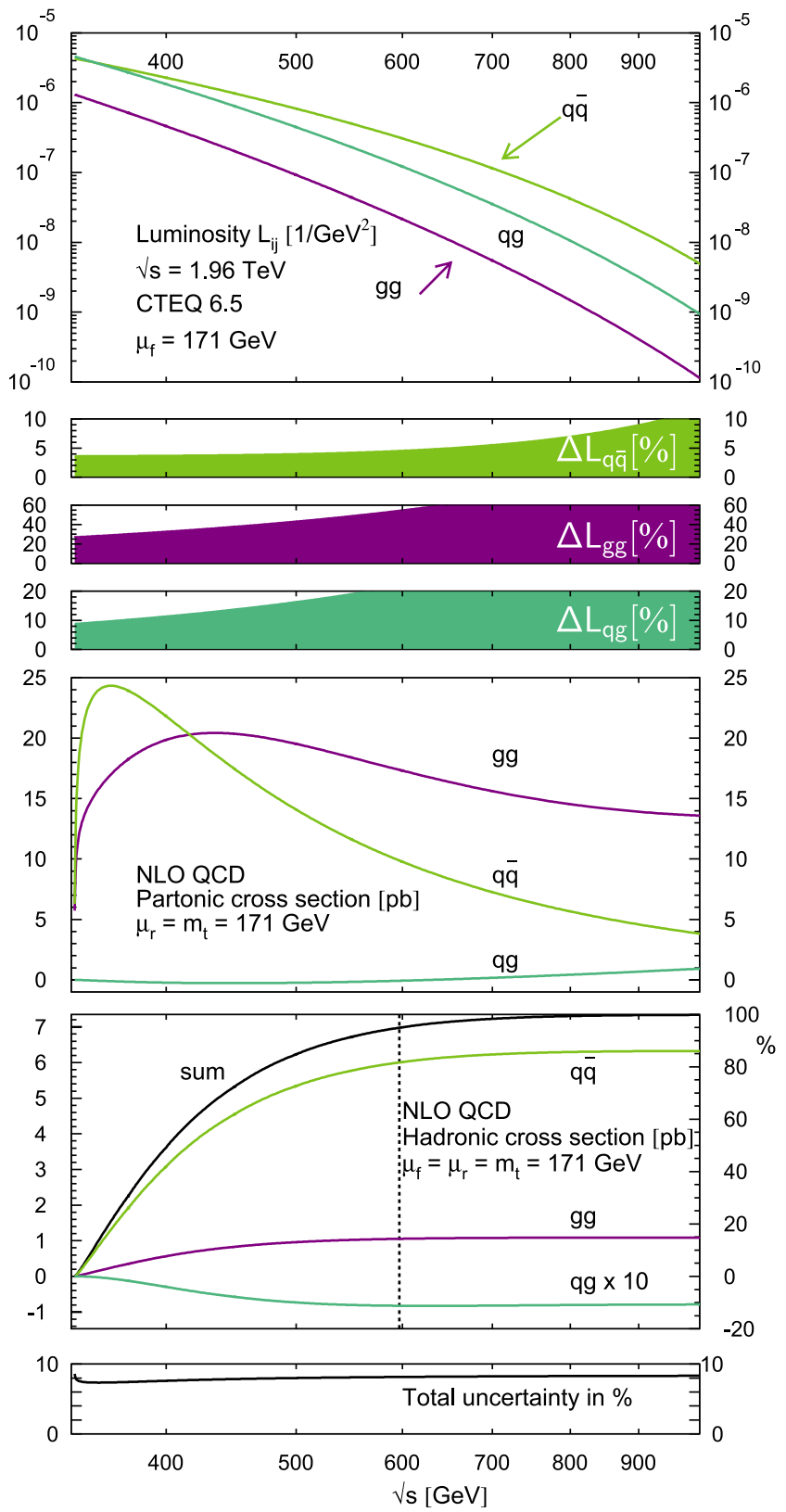

FIG. 2 (color online). Same as Fig. 1 for Tevatron $\left(\sqrt{s_{\text {had }}}=\right.$ $1.96 \mathrm{TeV})$ 
saturates the total cross section $\sigma\left(s_{\text {had }}, m_{t}^{2}\right)$ to $95 \%$. At Tevatron this happens at $\sqrt{s_{\max }} \approx 600 \mathrm{GeV}$ as can be seen from Fig. 2. Thus, the total cross section is largely dominated by parton kinematics in the range $\sqrt{\hat{s}} \approx 2 m_{t}$ close to the threshold of the top-quark pair. This makes topquark pair production at Tevatron an ideal place to apply threshold resummation. At LHC energies in contrast, the available phase space is larger and saturation to $95 \%$ is only reached at parton energies $\sqrt{s_{\max }} \approx 1 \mathrm{TeV}$ (see Fig. 1). This makes the cross section less sensitive to Sudakov logarithms, although numerically a significant part still originates from the threshold region for parton kinematics due to the steeply decreasing parton fluxes.

Further refinements of perturbative predictions for $\sigma_{p p \rightarrow t \bar{t} X}$ in Eq. (1) do rely on subsequent higher orders to be calculated. In particular, the knowledge about large logarithmic corrections from regions of phase space near partonic threshold allows for improvements of the theoretical accuracy beyond NLO in QCD. These Sudakovtype corrections can be organized to all orders by means of a threshold resummation (e.g. to NLL accuracy $[7,8]$ ), which has been the basis for phenomenological predictions employing a resummed cross section as defined in Ref. [8] (and also used in Ref. [14]).

However, before updating the NLL resummed results of Ref. [8], let us briefly give some relevant resummation formulas. It is well known that soft-gluon resummation for $t \bar{t}$ production relies on a decomposition of the partonlevel total cross section in the color basis, conveniently defined by color-singlet and color-octet final states. Then we can decompose

$$
\hat{\sigma}_{i j \rightarrow t \bar{t}}\left(\hat{s}, m_{t}^{2}, \mu_{f}^{2}, \mu_{r}^{2}\right)=\sum_{I=1,8} \hat{\sigma}_{i j, I}\left(\hat{s}, m_{t}^{2}, \mu_{f}^{2}, \mu_{r}^{2}\right) .
$$

Moreover, we use the standard definition of Mellin moments,

$$
\hat{\sigma}_{i j, I}^{N}\left(m_{t}^{2}, \mu_{f}^{2}, \mu_{r}^{2}\right)=\int_{0}^{1} d \rho \rho^{N-1} \hat{\sigma}_{i j, I}\left(\rho, m_{t}^{2}, \mu_{f}^{2}, \mu_{r}^{2}\right),
$$

with

$$
\rho=\frac{4 m_{t}^{2}}{\hat{s}} .
$$

Then, the resummed Mellin-space cross sections (defined in the $\overline{\mathrm{MS}}$ scheme) for the individual color structures of the scattering process are given by a single exponential (see e.g. Refs. $[22,23])$,

$$
\begin{aligned}
\frac{\hat{\sigma}_{i j, I}^{N}\left(m_{t}^{2}, \mu_{f}^{2}, \mu_{r}^{2}\right)}{\hat{\sigma}_{i j, I}^{(0), N}\left(m_{t}^{2}, \mu_{f}^{2}, \mu_{r}^{2}\right)}= & g_{i j, I}^{0}\left(m_{t}^{2}, \mu_{f}^{2}, \mu_{r}^{2}\right) \\
& \times \exp \left(G_{i j, I}^{N+1}\left(m_{t}^{2}, \mu_{f}^{2}, \mu_{r}^{2}\right)\right) \\
& +O\left(N^{-1} \ln ^{n} N\right),
\end{aligned}
$$

where $\hat{\sigma}_{i j, I}^{(0), N}$ denotes the Born term and the exponents $G_{i j, I}^{N}$ are commonly expressed as

$$
G_{i j I}^{N}=\ln N \cdot g_{i j}^{1}(\lambda)+g_{i j, I}^{2}(\lambda)+a_{s} g_{i j, I}^{3}(\lambda)+\cdots,
$$

where $\lambda=\beta_{0} a_{s} \ln N$ and $a_{s}=\alpha_{s} /(4 \pi)$. To NLL accuracy the (universal) functions $g_{i j}^{1}$ as well as the functions $g_{i j, I}^{2}$ are relevant in Eq. (10), of course, together with the appropriate matching functions $g_{i j, I}^{0}$ in Eq. (9). Explicit expressions can be found below.

For phenomenological applications [8], the soft-gluon resummation in $\mathrm{N}$-space at the parton level one introduces an improved (resummed) cross section $\sigma_{\text {res }}$, which is obtained by an inverse Mellin transformation as follows:

$$
\begin{aligned}
\sigma_{\operatorname{res}_{i j \rightarrow t t}}\left(\hat{s}, m_{t}^{2}, \mu_{f}^{2}, \mu_{r}^{2}\right)= & \int_{c-\mathrm{i} \infty}^{c+\mathrm{i} \infty} \frac{d N}{2 \pi \mathrm{i}} \rho^{-N+1} \\
& \times \sum_{I=1,8}\left(\hat{\sigma}_{i j, I}^{N}\left(m_{t}^{2}, \mu_{f}^{2}, \mu_{r}^{2}\right)\right. \\
& \left.-\left.\hat{\sigma}_{i j, I}^{N}\left(m_{t}^{2}, \mu_{f}^{2}, \mu_{r}^{2}\right)\right|_{\mathrm{NLO}}\right) \\
& +\hat{\sigma}_{i j \rightarrow t \bar{t}}^{\mathrm{NLO}}\left(\hat{s}_{t}, m_{t}^{2}, \mu_{f}^{2}, \mu_{r}^{2}\right),
\end{aligned}
$$

where $\hat{\sigma}_{i j \rightarrow t \bar{t}}^{\mathrm{NLO}}$ is the standard fixed order cross section at NLO in QCD and $\left.\hat{\sigma}_{i j, I}^{N}\right|_{\mathrm{NLO}}$ is the perturbative truncation of Eq. (9) at the same order in $\alpha_{s}$. Thus, the right-hand side of Eq. (11) reproduces the fixed order results and resums softgluon effects beyond NLO to NLL accuracy.

In Fig. 3 we plot the resummed cross section $\sigma_{\text {res }_{i j \rightarrow t \pi t}}$ as defined in Eq. (11) zooming in on the threshold region. We display the $q \bar{q}$ channel (left) and the $g g$ channel (right) as a function of the distance from the partonic threshold at $\sqrt{\hat{s}}=2 m_{t}$. As mentioned already the $q g$ channel is suppressed by an additional power of $\alpha_{s}$ and therefore does not contribute large Sudakov logarithms to the accuracy considered here.

The results for $\sigma_{\text {res }}$ as shown in Fig. 3 have been obtained by performing the inverse Mellin transform in Eq. (11) numerically. Following the procedure described in Ref. [8], we have compared our results obtained from the numerical inversion with the ones shown in Ref. [8] and found complete agreement. To be precise, the treatment of the constant terms in Ref. [8] [i.e. the terms denoted $g_{i j, I}^{0}$ in Eq. (9)] differs slightly from the minimal approach, e.g. Eq. (11). Some constants which are formally subleading have been included in Ref. [8], and moreover, several schemes for power suppressed terms in $N$ have been implemented. The resummed result shown in Fig. 3 is defined through Eq. (63) of Ref. [8] with the parameter $A$ set to 2. Another issue concerns the precise numerical matching of the exact NLO cross section and the resummed result in Eq. (11). We apply the resummed result only for $\sqrt{\hat{s}}-$ $2 m_{t} \leq 10 \mathrm{GeV}$. The exact point is determined from the crossing of the two curves for $\sigma_{\text {res }}$ and $\sigma_{\mathrm{NLO}}$. Note that the precise numerical value is not important. Effectively $\sigma_{\text {res }}$ is 

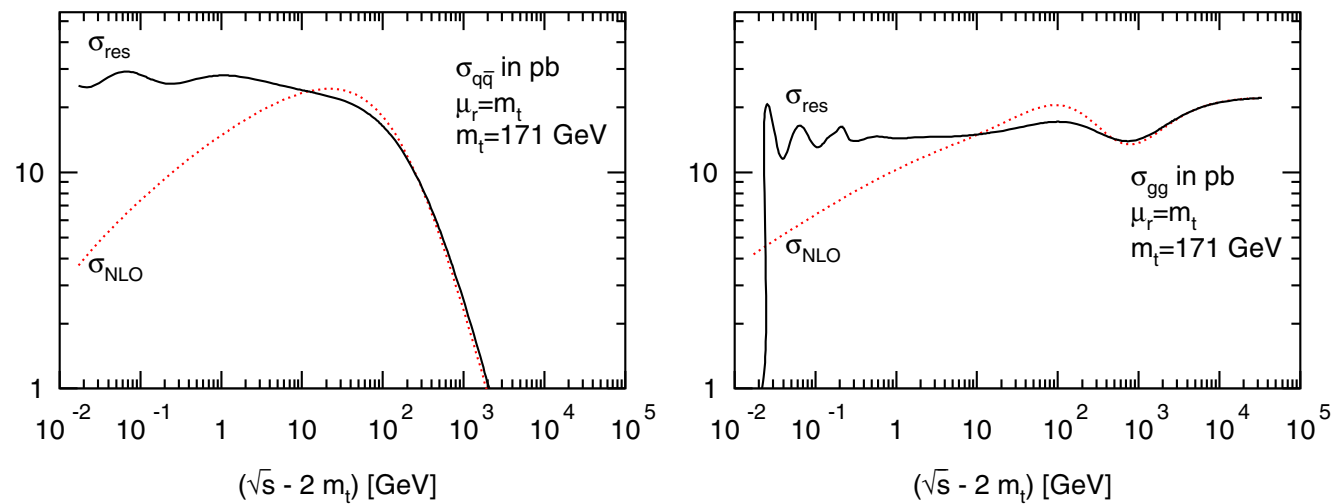

FIG. 3 (color online). The partonic cross sections for the processes $q \bar{q} \rightarrow t \bar{t}$ (left) and $g g \rightarrow t \bar{t}$ (right) in pb, and for $\mu=m_{t}=$ $171 \mathrm{GeV}$. The dotted lines are the exact NLO result [4,5] and the solid lines correspond to the NLL resummed cross sections [8] (see text for details).

TABLE I. The NLL resummed cross section of Ref. [8] in pb (see text for details) for various values of the top-quark mass $m_{t}$ at Tevatron $\left(\sqrt{s_{\text {had }}}=1.96 \mathrm{TeV}\right)$ using the CTEQ6.5 PDF set [24]. $\delta$ is the relative uncertainty with respect to the central value: $\delta=$ $100 \times(\max -\min ) /(\max +\min )$.

\begin{tabular}{|c|c|c|c|c|c|c|c|c|c|}
\hline \multirow[b]{2}{*}{$\mathrm{m}$} & \multicolumn{3}{|c|}{ Only scale uncertainty } & \multicolumn{3}{|c|}{ Only PDF uncertainty } & \multicolumn{3}{|c|}{ Total uncertainty } \\
\hline & $\min$ & $\max$ & $\delta[\%]$ & $\min$ & $\max$ & $\delta[\%]$ & $\min$ & $\max$ & $\delta[\%]$ \\
\hline 165 & 8.29 & 9.41 & 7 & 8.58 & 9.61 & 6 & 7.86 & 9.99 & 12 \\
\hline 166 & 8.03 & 9.11 & 7 & 8.31 & 9.3 & 6 & 7.61 & 9.67 & 12 \\
\hline 167 & 7.78 & 8.83 & 7 & 8.06 & 9.01 & 6 & 7.38 & 9.37 & 12 \\
\hline 168 & 7.54 & 8.55 & 7 & 7.81 & 8.73 & 6 & 7.15 & 9.07 & 12 \\
\hline 169 & 7.31 & 8.28 & 7 & 7.57 & 8.46 & 6 & 6.93 & 8.79 & 12 \\
\hline 170 & 7.09 & 8.03 & 7 & 7.34 & 8.2 & 6 & 6.72 & 8.51 & 12 \\
\hline 171 & 6.87 & 7.78 & 7 & 7.12 & 7.94 & 6 & 6.52 & 8.25 & 12 \\
\hline 172 & 6.66 & 7.54 & 7 & 6.9 & 7.7 & 6 & 6.32 & 7.99 & 12 \\
\hline 173 & 6.46 & 7.31 & 7 & 6.69 & 7.46 & 6 & 6.13 & 7.75 & 12 \\
\hline 174 & 6.26 & 7.09 & 7 & 6.49 & 7.23 & 6 & 5.94 & 7.51 & 12 \\
\hline 175 & 6.07 & 6.87 & 7 & 6.3 & 7.01 & 6 & 5.77 & 7.28 & 12 \\
\hline 176 & 5.89 & 6.67 & 7 & 6.11 & 6.8 & 6 & 5.59 & 7.06 & 12 \\
\hline 177 & 5.71 & 6.47 & 7 & 5.93 & 6.59 & 6 & 5.43 & 6.84 & 12 \\
\hline 178 & 5.54 & 6.27 & 7 & 5.75 & 6.4 & 6 & 5.26 & 6.64 & 12 \\
\hline 179 & 5.38 & 6.08 & 7 & 5.58 & 6.2 & 6 & 5.11 & 6.44 & 12 \\
\hline 180 & 5.22 & 5.9 & 7 & 5.41 & 6.02 & 6 & 4.96 & 6.24 & 12 \\
\hline
\end{tabular}

TABLE II. Same as in Table I using the MRST-2006 NNLO PDF set [25].

\begin{tabular}{|c|c|c|c|c|c|c|c|c|c|}
\hline \multirow[b]{2}{*}{$\underline{\mathrm{m}}$} & \multicolumn{3}{|c|}{ Only scale uncertainty } & \multicolumn{3}{|c|}{ Only PDF uncertainty } & \multicolumn{3}{|c|}{ Total uncertainty } \\
\hline & $\min$ & $\max$ & $\delta[\%]$ & $\min$ & $\max$ & $\delta[\%]$ & $\min$ & $\max$ & $\delta[\%]$ \\
\hline 165 & 8.53 & 9.87 & 8 & 9.19 & 9.7 & 3 & 8.32 & 10.1 & 10 \\
\hline 166 & 8.26 & 9.56 & 8 & 8.9 & 9.38 & 3 & 8.05 & 9.83 & 10 \\
\hline 167 & 8 & 9.25 & 8 & 8.62 & 9.08 & 3 & 7.8 & 9.51 & 10 \\
\hline 168 & 7.74 & 8.95 & 8 & 8.34 & 8.8 & 3 & 7.55 & 9.21 & 10 \\
\hline 169 & 7.5 & 8.67 & 8 & 8.08 & 8.52 & 3 & 7.31 & 8.92 & 10 \\
\hline 170 & 7.26 & 8.4 & 8 & 7.83 & 8.25 & 3 & 7.08 & 8.63 & 10 \\
\hline 171 & 7.04 & 8.13 & 8 & 7.58 & 7.99 & 3 & 6.86 & 8.36 & 10 \\
\hline 172 & 6.82 & 7.87 & 8 & 7.35 & 7.74 & 3 & 6.65 & 8.1 & 10 \\
\hline 173 & 6.6 & 7.63 & 8 & 7.12 & 7.5 & 3 & 6.44 & 7.84 & 10 \\
\hline 174 & 6.4 & 7.39 & 8 & 6.9 & 7.26 & 3 & 6.24 & 7.6 & 10 \\
\hline 175 & 6.2 & 7.16 & 8 & 6.69 & 7.04 & 3 & 6.05 & 7.36 & 10 \\
\hline 176 & 6.01 & 6.94 & 8 & 6.48 & 6.82 & 3 & 5.87 & 7.13 & 10 \\
\hline 177 & 5.83 & 6.73 & 8 & 6.28 & 6.61 & 3 & 5.69 & 6.91 & 10 \\
\hline 178 & 5.65 & 6.52 & 8 & 6.09 & 6.41 & 3 & 5.51 & 6.7 & 10 \\
\hline 179 & 5.48 & 6.32 & 8 & 5.9 & 6.21 & 3 & 5.35 & 6.49 & 10 \\
\hline 180 & 5.31 & 6.13 & 8 & 5.72 & 6.02 & 3 & 5.18 & 6.29 & 10 \\
\hline
\end{tabular}


TABLE III. The NLL resummed cross section of Ref. [8] in pb (see text for details) for various values of the top-quark mass $m_{t}$ at LHC $\left(\sqrt{s_{\text {had }}}=14 \mathrm{TeV}\right)$ using the CTEQ6.5 PDF set [24].

\begin{tabular}{|c|c|c|c|c|c|c|c|c|c|}
\hline \multirow[b]{2}{*}{$\mathrm{m}$} & \multicolumn{3}{|c|}{ Only scale uncertainty } & \multicolumn{3}{|c|}{ Only PDF uncertainty } & \multicolumn{3}{|c|}{ Total uncertainty } \\
\hline & $\min$ & $\max$ & $\delta[\%]$ & $\min$ & $\max$ & $\delta[\%]$ & $\min$ & $\max$ & $\delta[\%]$ \\
\hline 165 & 937 & 1154 & 11 & 1006 & 1074 & 4 & 906 & 1191 & 14 \\
\hline 166 & 911 & 1122 & 11 & 978 & 1044 & 4 & 881 & 1159 & 14 \\
\hline 167 & 886 & 1091 & 11 & 951 & 1016 & 4 & 856 & 1127 & 14 \\
\hline 168 & 862 & 1061 & 11 & 925 & 988 & 4 & 833 & 1096 & 14 \\
\hline 169 & 838 & 1032 & 11 & 900 & 962 & 4 & 810 & 1066 & 14 \\
\hline 170 & 816 & 1004 & 11 & 875 & 936 & 4 & 788 & 1038 & 14 \\
\hline 171 & 794 & 977 & 11 & 852 & 911 & 4 & 767 & 1010 & 14 \\
\hline 172 & 773 & 950 & 11 & 829 & 887 & 4 & 746 & 983 & 14 \\
\hline 173 & 752 & 925 & 11 & 806 & 863 & 4 & 726 & 957 & 14 \\
\hline 174 & 732 & 900 & 11 & 785 & 841 & 4 & 707 & 931 & 14 \\
\hline 175 & 713 & 877 & 11 & 764 & 819 & 4 & 688 & 907 & 14 \\
\hline 176 & 694 & 853 & 11 & 744 & 797 & 4 & 670 & 883 & 14 \\
\hline 177 & 676 & 831 & 11 & 724 & 777 & 4 & 653 & 860 & 14 \\
\hline 178 & 659 & 809 & 11 & 705 & 757 & 4 & 636 & 838 & 14 \\
\hline 179 & 642 & 788 & 11 & 687 & 737 & 4 & 619 & 816 & 14 \\
\hline 180 & 625 & 768 & 11 & 669 & 718 & 4 & 603 & 795 & 14 \\
\hline
\end{tabular}

thereby restricted to a region of parton energies of $\sqrt{\hat{s}} \approx$ $2 m_{t}$ or, in other words, to a kinetic energy of one top quark of a few GeV.

It should be stressed that the total hadronic cross section is not very sensitive to these fine details due to the convolution with the parton luminosities $L_{i j}$ in Eq. (1). However, to per mille accuracy (see Tables I, II, III, and IV) these details become noticeable. Having them clarified, we are now in a position to update previous results $[8,14]$ for the $t \bar{t}$ cross section using modern PDFs, such as the CTEQ6.5 PDF set [24]. In comparison to older sets, we find e.g. a shift of $3 \%$ in the total cross section between the PDF sets CTEQ6.5 [24] and CTEQ6.1 [21], see also Ref. [11]. Since Eq. (11) effectively contains the dominant part of higher orders (NNLO and beyond), it seems however equally appropriate to use also the MRST-2006 NNLO PDF set [25]. From the results in Tables I, II, III, and IV and Fig. 4, we conclude that the present overall uncertainty on the NLL resummed $t \bar{t}$ cross section is $12 \%$ at Tevatron with a small shift of the central value between different PDF sets. At LHC the NLL resummed cross section of Ref. [8] reduces effectively to the NLO QCD prediction of Refs. $[4,5]$ with an overall uncertainty of $14 \%$. This is due to the small gluon contribution close to threshold (see

TABLE IV. Same as in Table III using the MRST-2006 NNLO PDF set [25].

\begin{tabular}{|c|c|c|c|c|c|c|c|c|c|}
\hline \multirow[b]{2}{*}{$\mathrm{m}$} & \multicolumn{3}{|c|}{ Only scale uncertainty } & \multicolumn{3}{|c|}{ Only PDF uncertainty } & \multicolumn{3}{|c|}{ Total uncertainty } \\
\hline & $\min$ & $\max$ & $\delta[\%]$ & $\min$ & $\max$ & $\delta[\%]$ & $\min$ & $\max$ & $\delta[\%]$ \\
\hline 165 & 986 & 1222 & 11 & 1084 & 1110 & 2 & 974 & 1236 & 12 \\
\hline 166 & 959 & 1189 & 11 & 1054 & 1080 & 2 & 947 & 1203 & 12 \\
\hline 167 & 933 & 1156 & 11 & 1026 & 1050 & 2 & 921 & 1170 & 12 \\
\hline 168 & 908 & 1125 & 11 & 998 & 1022 & 2 & 896 & 1138 & 12 \\
\hline 169 & 883 & 1094 & 11 & 971 & 995 & 2 & 872 & 1108 & 12 \\
\hline 170 & 860 & 1065 & 11 & 945 & 968 & 2 & 849 & 1078 & 12 \\
\hline 171 & 837 & 1036 & 11 & 920 & 943 & 2 & 826 & 1049 & 12 \\
\hline 172 & 815 & 1009 & 11 & 895 & 918 & 2 & 804 & 1021 & 12 \\
\hline 173 & 793 & 982 & 11 & 872 & 894 & 2 & 783 & 994 & 12 \\
\hline 174 & 773 & 956 & 11 & 849 & 870 & 2 & 763 & 968 & 12 \\
\hline 175 & 753 & 931 & 11 & 827 & 848 & 2 & 743 & 943 & 12 \\
\hline 176 & 733 & 907 & 11 & 805 & 826 & 2 & 723 & 918 & 12 \\
\hline 177 & 714 & 883 & 11 & 784 & 805 & 2 & 705 & 895 & 12 \\
\hline 178 & 696 & 860 & 11 & 764 & 784 & 2 & 686 & 872 & 12 \\
\hline 179 & 678 & 838 & 11 & 744 & 764 & 2 & 669 & 849 & 12 \\
\hline 180 & 661 & 817 & 11 & 725 & 745 & 2 & 652 & 828 & 12 \\
\hline
\end{tabular}



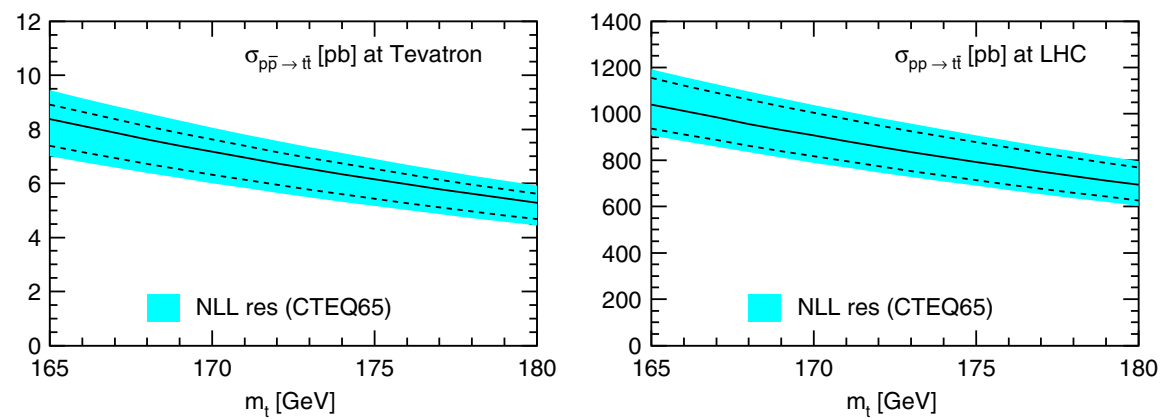

FIG. 4 (color online). The $t \bar{t}$ total cross section resummed to NLL accuracy [8] as a function of $m_{t}$ for the Tevatron at $\sqrt{s_{\text {had }}}=$ $1.96 \mathrm{TeV}$ (left) and LHC at $\sqrt{s_{\text {had }}}=14 \mathrm{TeV}$ (right). The solid line is the central value for $\mu=m_{t}$, the dashed lower and upper lines correspond to $\mu=2 m_{t}$ and $\mu=m_{t} / 2$, respectively. The band denotes the total uncertainty that is the uncertainty due to scale variations and the PDF uncertainty of the CTEQ6.5 set [24] combined together according to Eq. (4).

Fig. 3) and the cut when matching NLO and the $\sigma_{\text {res }}$ in the numerical determinations. Thus, the reduction of the theoretical uncertainty through Eq. (11) is marginal in this case. We will see in Sec. III, how this situation can be improved with the help of approximate NNLO QCD corrections.

\section{PROSPECTS AT NNLO IN QCD}

Let us now extend the theory predictions for heavyquark hadroproduction. We will focus on the threshold region and improve soft-gluon resummation to NNLL accuracy. Subsequently, we employ the resummed cross section to generate higher order perturbative correctionsmore specifically an approximate NNLO cross section $\sigma_{\mathrm{NNLO}(\text { approx) }}$ which is exact to logarithmic accuracy (including the Coulomb corrections). To that end, we briefly recall the steps leading to the final form for $G_{i j, I}^{N}$ in Eq. (10). In order to achieve NNLL accuracy the function $g_{i j, I}^{3}$ is of particular interest here.

The exponential $G_{i j, I}^{N}$ in Eq. (9) is built up from universal radiative factors for the individual color structures which take the form

$$
G_{q \bar{q} / g g, I}^{N}=G_{\mathrm{DY} / \mathrm{Higgs}}^{N}+\delta_{I, 8} G_{Q \bar{Q}}^{N},
$$

where the exponentiation of singlet contribution (i.e. a colorless massive final state) follows from the Drell-Yan (DY) process and hadronic Higgs production in gluon fusion. The corresponding functions $G_{\mathrm{DY}}^{N}$ and $G_{\mathrm{Higgs}}^{N}$ are very well known [26-28]. The exponentiation of the coloroctet contribution receives an additional contribution $G_{Q \bar{Q}}^{N}$ due to soft-gluon emission from the heavy-quark pair in the final state. The final-state system carries a total color charge given by the $Q \bar{Q}$ charge, thus its contribution to soft radiation vanishes in the color-singlet channels regardless of the initial-state partons. Moreover, gluon emission from massive quarks does not lead to collinear logarithms and therefore $G_{Q \bar{Q}}^{N}$ starts at NLL accuracy only. Explicit formulas are

$$
\begin{aligned}
G_{\mathrm{DY} / \mathrm{Higgs}}^{N}= & \int_{0}^{1} d z \frac{z^{N-1}-1}{1-z} \int_{\mu_{f}^{2}}^{4 m_{t}^{2}(1-z)^{2}} \frac{d q^{2}}{q^{2}} 2 A_{i}\left(\alpha_{s}\left(q^{2}\right)\right) \\
& +D_{i}\left(\alpha_{s}\left(4 m_{t}^{2}[1-z]^{2}\right)\right) \\
G_{Q \bar{Q}}^{N}= & \int_{0}^{1} d z \frac{z^{N-1}-1}{1-z} D_{Q \bar{Q}}\left(\alpha_{s}\left(4 m_{t}^{2}[1-z]^{2}\right)\right)
\end{aligned}
$$

with anomalous dimensions $A_{i}$ and $D_{i}, i=q, g$ corresponding to DY and Higgs, respectively. The effects of collinear soft-gluon radiation off initial-state partons $i=$ $q, g$ are collected by the first term in Eq. (13) while the process-dependent contributions from large-angle soft gluons are resummed by the second term. Soft radiation from the heavy-quark pair in the final state is summarized by $G_{Q \bar{Q}}^{N}$ in Eq. (14) with the corresponding anomalous dimension $D_{Q \bar{Q}}$.

The extension to NNLL requires the $A_{q}$ and $A_{g}$ to three loops [29,30] and the function $D_{q}$ and $D_{g}$ to two loops [2628] (the latter are actually known to three loops as well [31,32]). Explicit expressions using the expansions

$$
f\left(\alpha_{s}\right)=\sum_{l} f^{(l)} \frac{\alpha_{s}^{l}}{4 \pi} \equiv \sum_{l} f^{(l)} a_{s}^{l} .
$$

are collected in Eqs. (A2)-(A4) in Appendix A. The remaining anomalous dimensions $D_{Q \bar{Q}}$ for soft radiation off a heavy-quark pair in the final state are needed to two loops. For the latter we use the exact calculation of the two-loop QCD corrections to the massive heavy-quark form factor [33] along with Ref. [34], where the exponentiation of the form factor for massive colored particles in the limit $m^{2} \rightarrow 0$ has been clarified. From the all-order singularity structure of the massive form factor [34], we can read off the single poles corresponding to soft-gluon emission at one and two loops. After a trivial substitution of color factors $\left(C_{A}\right.$ for $\left.C_{F}\right)$, we find

$$
D_{Q \bar{Q}}^{(1)}=-A_{g}^{(1)}, \quad D_{Q \bar{Q}}^{(2)}=-A_{g}^{(2)} .
$$


As pointed out in Ref. [8], the one-loop value of $D_{Q \bar{Q}}$ agrees with Ref. [7] where the soft anomalous dimension matrix (in color space) $\Gamma_{I J}^{(1)}$ for heavy-quark production has been calculated at order $\alpha_{s}$. In the limit $\beta \rightarrow 0$ the matrix $\Gamma_{I J}^{(1)}$ diagonalizes in the singlet-octet basis and reproduces $D_{Q \bar{Q}}^{(1)}$ from its eigenvalue in the octet channel. Moreover, the structure of $D_{Q \bar{Q}}$ as determined from Ref. [34] agrees also with the two-loop soft anomalous dimension matrix $\Gamma_{I J}^{(2)}$ for vanishing parton masses which obeys the following factorization property $[35,36]$ :

$$
\left.\Gamma_{I J}\right|_{m=0}=\left.a_{s} \Gamma_{I J}^{(1)}\right|_{m=0}\left(1+a_{s} \frac{A_{g}^{(2)}}{A_{g}^{(1)}}\right),
$$

with the well-known ratio $A_{g}^{(2)} / A_{g}^{(1)}$ [37]. As a further check on Eq. (16) it would, of course, be very interesting to repeat the calculation of Refs. $[35,36]$ for heavy-quark hadroproduction at two loops, i.e. with nonvanishing parton masses $m \neq 0$.

Finally, explicit integration of Eqs. (13) and (14) leads to the functions $g_{i j}^{1}, g_{i j, I}^{2}, g_{i j, I}^{3}$ of Eq. (10) and to the matching $g_{i j, I}^{0}$ in Eq. (9) which we collected in Eqs. (A5)-(A13) in Appendix A. All formulas can be obtained by simple substitutions e.g. from deep-inelastic scattering (DIS) in Ref. [28]. In Fig. 5 we display the resummed cross section of Eq. (9) (normalized to the respective Born result) for the $q \bar{q}$ and the $g g$ channel in increasing logarithmic accuracy. Figure 5 clearly shows the good convergence property of the NNLL contribution similar to other observables investigated previously [26-28]. As a matter of fact, Eq. (10) may even be extended to next-to-next-to-next-to-leading accuracy $\left(\mathrm{N}^{3} \mathrm{LL}\right)$, as the relevant functions $g_{i j, I}^{4}$ can be easily derived from Ref. [28] and the respective anomalous dimensions are known. Following the arguments based on the exponentiation of the form factor for massive colored partons [34] leading to Eq. (16), we identify $D_{Q \bar{Q}}^{(3)}=-A_{g}^{(3)}$ and for the four-loop terms $A_{q}^{(4)}$ and $A_{g}^{(4)}$ a Padé estimate exists [28]. However, presently we are lacking knowledge on the matching functions $g_{i j}^{0}$ at this order. From experience we expect $\mathrm{N}^{3} \mathrm{LL}$ effects to be numerically very small, though, and we leave this issue to future investigations.

Let us instead use the resummed cross section $\sigma_{\text {res }}$ (now known at NNLL accuracy) to construct an approximate NNLO cross section $\sigma_{\mathrm{NNLO}}$ (approx) by expanding Eq. (9) to second order. In this way, we determine the corresponding Sudakov logarithms appearing in the NNLO corrections, i.e. the powers of $\ln ^{k} N$ in Mellin space or $\ln ^{k} \beta$ in momentum space with $k=1, \ldots, 4$ and the velocity of the heavy quark

$$
\beta=\sqrt{1-4 m_{t}^{2} / s}
$$

As a technical remark, we stress here that in a fixed order expansion the inverse Mellin transformation, i.e. the mapping of powers of $\ln N$ to powers of $\ln (\beta)$ can be uniquely performed. The Mellin-space accuracy up to power suppressed terms in $N$ corresponds to neglecting higher order polynomials in $(1-\rho)$ with $\rho=4 m_{t}^{2} / \hat{s}$. We give some formulas in Appendix A. Moreover, we can even include the complete Coulomb corrections at two loops thanks to Refs. [33,38]. In the singlet-octet decomposition $\hat{\sigma}_{i j, I}$ of the individual color structures in the cross section, the result of Refs. $[33,38]$ can be directly applied to the singlet case, while a simple modification of the color factors [that is $\left(C_{F}-C_{A} / 2\right)$ instead of $\left.C_{F}\right]$ accounts for the octet case (see e.g. [39]).

Thus, we are in a position to present the threshold expansion of the inclusive partonic cross sections $\hat{\sigma}_{i j \rightarrow t \bar{t}}\left(s, m^{2}, \mu^{2}\right)$ entering Eq. (1). In the perturbative expansions in powers of the strong coupling constant $\alpha_{s}$ as defined in Eq. (15) and setting $\mu=m_{t}$ and $n_{f}=5$, we have for the $q \bar{q}$ channel in the $\overline{\mathrm{MS}}$ scheme

$$
\begin{aligned}
\hat{\sigma}_{q \bar{q} \rightarrow t \bar{t}}^{(1)}= & \hat{\sigma}_{q \bar{q} \rightarrow t \bar{t}}^{(0)}\left\{42.667 \ln ^{2} \beta-20.610 \ln \beta+13.910\right. \\
& \left.-3.2899 \frac{1}{\beta}\right\},
\end{aligned}
$$
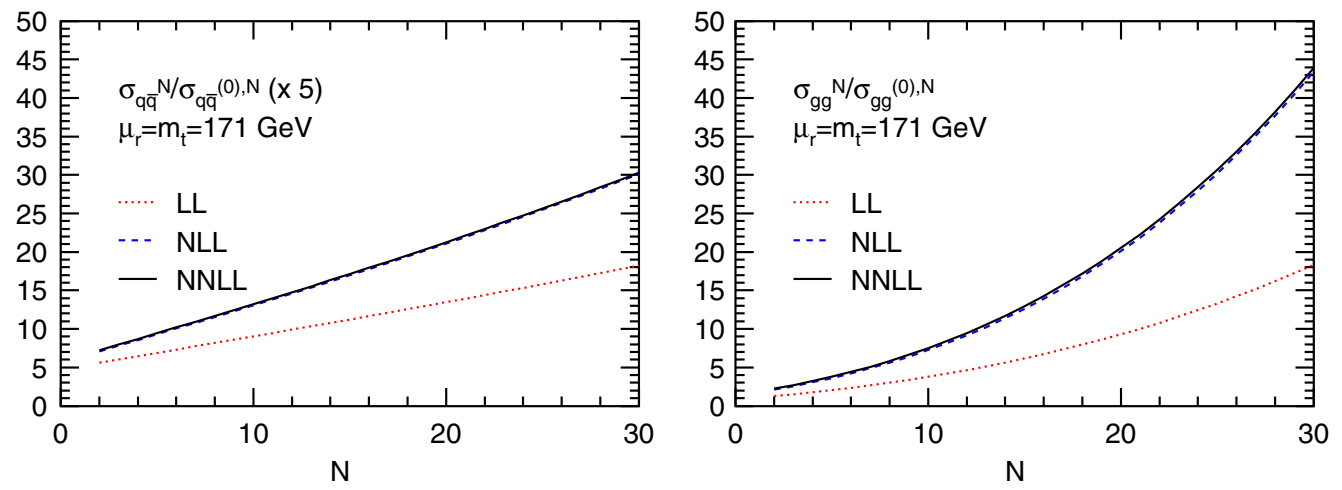

FIG. 5 (color online). The resummed cross section in Mellin space for the $q \bar{q}$ (left) and the $g g$ channel (right) normalized to the Born result for $\mu=m_{t}=171 \mathrm{GeV}$. The dotted lines are the LL approximation, the dashed lines denote the NLL result, and the solid lines correspond to the NNLL result derived in this paper. 


$$
\begin{aligned}
\hat{\sigma}_{q \bar{q} \rightarrow t \bar{t}}^{(2)}= & \hat{\sigma}_{q \bar{q} \rightarrow t i \bar{t}}^{(0)}\left\{910.22 \ln ^{4} \beta-1315.5 \ln ^{3} \beta+(565.80\right. \\
& \left.-140.37 \frac{1}{\beta}\right) \ln ^{2} \beta+\left(862.42+32.106 \frac{1}{\beta}\right) \ln \beta \\
& \left.+3.6077 \frac{1}{\beta^{2}}+10.474 \frac{1}{\beta}+C_{q \bar{q}}^{(2)}\right\}, \\
\hat{\sigma}_{g g \rightarrow t \bar{t}}^{(1)}= & \hat{\sigma}_{g g \rightarrow t \bar{t}}^{(0)}\left\{96 \ln ^{2} \beta-9.5165 \ln \beta+35.322\right. \\
& \left.+5.1698 \frac{1}{\beta}\right\}, \\
\hat{\sigma}_{g g \rightarrow t \bar{t}}^{(2)}= & \hat{\sigma}_{g g \rightarrow t \bar{t}}^{(0)}\left\{4608 \ln ^{4} \beta-1894.9 \ln ^{3} \beta+(-3.4811\right. \\
& \left.+496.30 \frac{1}{\beta}\right) \ln ^{2} \beta+\left(3144.4+321.17 \frac{1}{\beta}\right) \ln \beta \\
& \left.+68.547 \frac{1}{\beta^{2}}-196.93 \frac{1}{\beta}+C_{g g}^{(2)}\right\},
\end{aligned}
$$

The terms proportional to inverse powers of $\beta$ correspond to the Coulomb corrections and the presently unknown two-loop constants $C_{q \bar{q}}^{(2)}$ and $C_{g g}^{(2)}$ are set to zero. For reference, we have also repeated the well-known NLO results [Eqs. (18) and (20)]. The lengthy analytical results (containing the explicit dependence on the color factors $C_{A}, C_{F}$ and on $n_{f}$ ) are given in Appendix A, Eqs. (A17)-(A20). We define a NNLO (approximate) cross section to be used in this paper as the sum of exact NLO result and the two-loop contribution of Eqs. (19) and (21) for all scale independent terms. All scale dependent terms at NNLO accuracy are long known exactly [9] as they can be easily constructed from the lower orders convoluted with the appropriate splitting functions. We use the exact result of Ref. [9] for the $\mu$ dependence at two loops. As emphasized several times, the $q g$ and $\bar{q} g$ contributions are small at Tevatron and LHC and we simply keep them at NLO here.

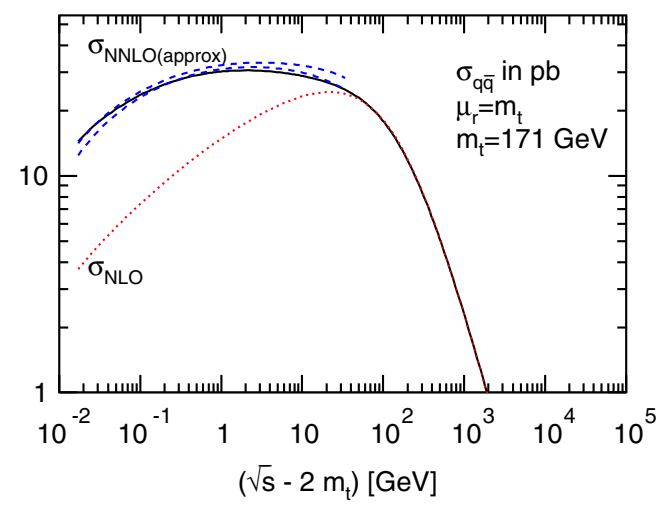

In Fig. 6 we display the NNLO (approximate) results for the partonic cross sections as calculated in Eqs. (19) and (21). We plot $\hat{\sigma}_{q \bar{q} \rightarrow t \bar{t}}$ (left) and $\hat{\sigma}_{g g \rightarrow t \bar{t}}$ (right) again as a function of the distance from threshold at $\sqrt{\hat{s}}=2 m_{t}$ (solid lines in Fig. 6). This is useful to assess the question of what improvements can be expected from a full NNLO calculation. Given that the $K$ factor of the NLO result is of moderate size at large partonic energies, one can expect that the full NNLO corrections should give only small corrections in this region of phase space. On the other hand, the $K$ factor of the NLO correction becomes large in the threshold region [i.e. $\sqrt{\hat{s}}-2 m_{t} \lesssim O(50) \mathrm{GeV}$ ], where finally perturbation theory breaks down. In this region the corrections are dominated by the large logarithms in $\beta$ together with the Coulomb corrections which are correctly described by Eqs. (19) and (21). In other words, where we expect large corrections from the full NNLO QCD calculation our $\sigma_{\mathrm{NNLO}}$ (approx) should provide a good estimate. This is further supported by Ref. [15] where the next-to-leading order corrections to top-quark pair production together with an additional jet have been calculated. This contribution represents part of the NNLO corrections for inclusive top-quark pair production. In Ref. [15] it was found that for $\mu=\mu_{f}=\mu_{r}=m_{t}$ the NLO corrections to $t \bar{t}+1-$ jet production are almost zero. This is a further indication that the hard corrections to the inclusive top-quark pair production at NNLO are indeed small. Moreover, as mentioned above, we have further improved $\sigma_{\mathrm{NNLO} \text { (approx) }}$ by incorporating the complete scale dependence at NNLO which is already known exactly [9]. To that end, let us quantify in detail once more the range of validity for the soft-gluon approximations. We show in Table $\mathrm{V}$ the numerical size of the individual logarithms and powers in $\beta$ in Eqs. (18), (20), and (21) when evaluated at a given parton energy $\hat{s}$. As a consequence of the moderate size of the expansion coefficients in Eqs. (18), (20), and (21), we clearly see the good convergence properties of the logarithmic expansion up

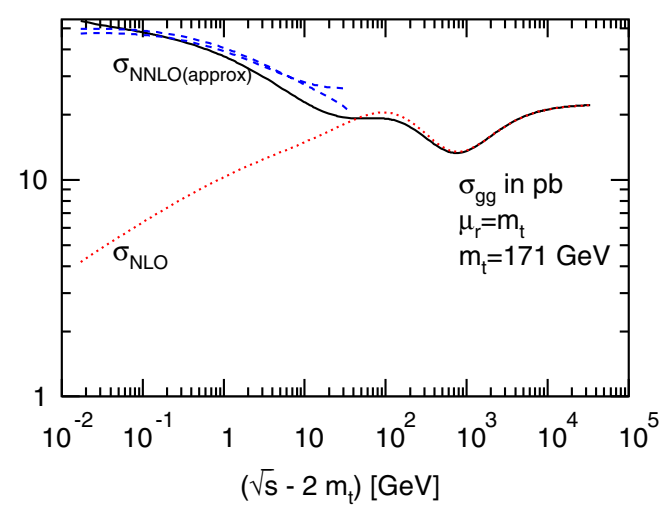

FIG. 6 (color online). The partonic cross sections for the processes $q \bar{q} \rightarrow t \bar{t}$ (left) and $g g \rightarrow t \bar{t}$ (right) in pb, and for $\mu=m_{t}=$ $171 \mathrm{GeV}$. The dotted lines are the exact NLO result [4,5] and the solid lines correspond to the NNLO (approximate) result of this paper. The dashed lines close by denote the previous approximations of [9]. 
TABLE V. Numerical values of the individual powers of $\ln \beta$ and $\beta$ for various distances from threshold $\sqrt{\hat{s}}-2 m_{t}$ for $m_{t}=$ $171 \mathrm{GeV}$ as entering in Eqs. (18)-(21).

\begin{tabular}{|c|c|c|c|c|c|c|c|}
\hline$\sqrt{\hat{S}}-2 m_{t}[\mathrm{GeV}]$ & $\ln ^{4} \beta$ & $\ln ^{3} \beta$ & $\ln ^{2} \beta$ & $\ln \beta$ & $\beta^{-2}$ & $\beta^{-1}$ & $\beta$ \\
\hline 0.1 & 191.9844 & -51.5762 & 13.8558 & -3.7223 & 1710.7500 & 41.3612 & 0.0242 \\
\hline 0.5 & 72.5503 & -24.8588 & 8.5176 & -2.9185 & 342.7502 & 18.5135 & 0.0540 \\
\hline 1.0 & 43.8302 & -17.0345 & 6.6204 & -2.5730 & 171.7504 & 13.1054 & 0.0763 \\
\hline 5.0 & 9.9709 & -5.6111 & 3.1577 & -1.7770 & 34.9518 & 5.9120 & 0.1691 \\
\hline 10.0 & 4.3130 & -2.9928 & 2.0768 & -1.4411 & 17.8536 & 4.2254 & 0.2367 \\
\hline 50.0 & 0.2628 & -0.3671 & 0.5127 & -0.7160 & 4.1870 & 2.0462 & 0.4887 \\
\hline 100.0 & 0.0434 & -0.0951 & 0.2084 & -0.4565 & 2.4919 & 1.5786 & 0.6335 \\
\hline 500.0 & 0.0001 & -0.0007 & 0.0081 & -0.0901 & 1.1976 & 1.0943 & 0.9138 \\
\hline
\end{tabular}

energies $\sqrt{\hat{s}}-2 m_{t} \lesssim O(50) \mathrm{GeV}$. Beyond that value the soft logarithms (being proportional to the Born cross section) smoothly vanish.

In addition we show in Fig. 6 also previous approximations to the NNLO correction from Ref. [9] (dashed lines) employing two distinct differential kinematics to define the partonic threshold. They agree with our NNLO (approximate) corrections for $\sqrt{\hat{s}}-2 m_{t} \lesssim 30 \mathrm{GeV}$ if Eqs. (19) and (21) are truncated to the first three powers in $\ln \beta$. However, at higher partonic center-of-mass energies, the results of Ref. [9] receive large numerical contributions from subleading terms and become unreliable (see, in particular, Fig. 6 on the right).

We would also like to point out that there is a discrepancy between the NLL resummed cross section of Ref. [8] and Eq. (11) and the fixed order NNLO approximation discussed here. In particular, for the gluon fusion channel in Fig. 3 (right) and Fig. 6 (right), the numerical differences between $\sigma_{\text {res }}$ and $\sigma_{\mathrm{NNLO} \text { (approx) }}$ are rather large. The allorder NLL resummed cross section $\sigma_{\text {res }}$ is significantly smaller than its expansion to second-order Eq. (21) or the corresponding result of Ref. [9], the latter two both being consistent with each other. We can attribute this difference to the following fact: For $t \bar{t}$-hadroproduction the Born cross section exhibits simple (although nontrivial) $N$ dependence and the resummed cross section $\sigma_{\text {res }}$ in Mellin space [as implemented in Ref. [8] and Eq. (11)] contains products of $N$-dependent functions. This is unlike other cases considered in the literature, where the Born terms have always been proportional to a delta function, i.e. $\delta(1-x)$ for DIS, Drell-Yan, or Higgs production. In momentum space products of $N$-dependent functions correspond to convolutions, which induce formally subleading but numerically large corrections in the resummed result. Eventually, this leads to the observed suppression in Fig. 3. Most likely this large discrepancy will also persist when comparing $\sigma_{\text {res }}$ to a full NNLO QCD calculation, or upon matching the latter to a resummed cross section at NNLL accuracy along the lines of Eq. (11). This fact has to be kept in mind when using $\sigma_{\text {res }}$ for predictions at LHC, where the $g g$ channel dominates. As mentioned above, for any finite-order expansion in $\alpha_{s}$, there is no ambiguity in performing the inverse Mellin transformation analytically up to power suppressed terms in $N$ or, equivalently in $(1-\rho)$.

We are now in a position to present the new results for the top-quark cross section $\sigma_{p p \rightarrow t \bar{t} X}$ at NNLO (approximate) as defined below Eq. (21) including the exact scale dependence. We also quote the corresponding uncertainty according to Eq. (4). In our study we use the same PDFs as in Tables I, II, III, and IV above. In Fig. 7 the scale dependence for $\sigma_{\mathrm{NLO}}$ and $\sigma_{\mathrm{NNLO} \text { (approx) }}$ is shown. For the $\sigma_{\text {NNLO(approx) }}$ we use the PDF set MRST-2006 NNLO while for $\sigma_{\mathrm{NLO}}$ the PDF set CTEQ6.5 is used. To become less sensitive to different normalizations, we normalize the curves to the central value $\sigma\left(\mu=m_{t}\right)$. In addition we show also the results obtained by using the Alekhin PDF set [40] available in NLO and NNLO accuracy. After normalization one can see that the two curves for the NLO predictions agree rather well as one might expect. For the two NNLO curves the agreement is less good, in particular, for extreme values of the scale. The origin of the

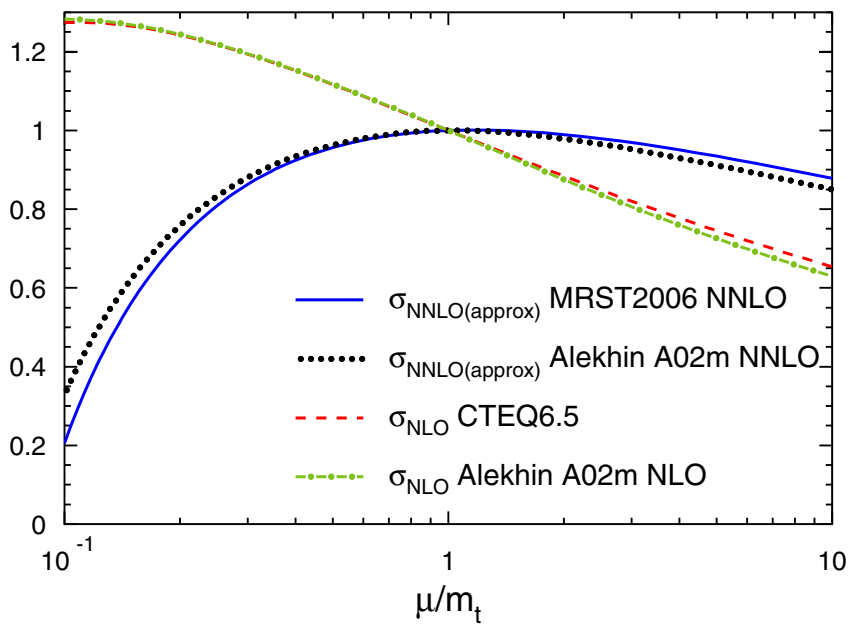

FIG. 7 (color online). Comparison of the scale dependence of $\sigma_{\mathrm{NNLO}(\text { approx) }}$ with PDF set MRST-2006 NNLO [25] and $\sigma_{\mathrm{NLO}}$ with PDF set CTEQ6.5 [24]. For comparison we show also the corresponding results for the Alekhin set of PDFs [40]. The cross sections are normalized to the value at $\mu=m_{t}$. 

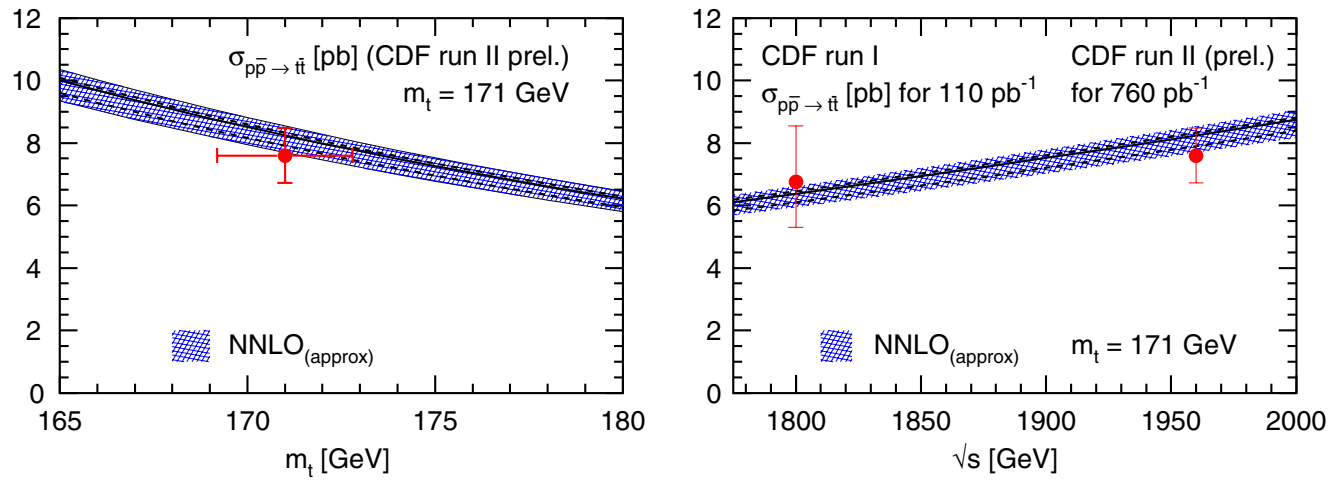

FIG. 8 (color online). The NNLO (approximate) QCD prediction for the $t \bar{t}$ total cross section at Tevatron and CDF data [41] with $m_{t}=171 \mathrm{GeV}$-as functions of $m_{t}$ for $\sqrt{s_{\text {had }}}=1.96 \mathrm{TeV}$ (left) and of $\sqrt{s_{\text {had }}}$ (right). The solid line is the central value for $\mu=m_{t}$, the dashed lower and upper lines correspond to $\mu=2 m_{t}$ and $\mu=m_{t} / 2$, respectively. The band denotes the total uncertainty that is the uncertainty due to scale variations and the PDF uncertainty of the MRST-2006 NNLO set [25] combined together according to Eq. (4).

TABLE VI. The cross section $\sigma_{\mathrm{NNLO} \text { (approx) }}$ as derived in this paper in pb for various values of the top-quark mass $m_{t}$ at Tevatron $\left(\sqrt{s_{\text {had }}}=1.96 \mathrm{TeV}\right)$ using the CTEQ6.5 PDF set [24].

\begin{tabular}{|c|c|c|c|c|c|c|c|c|c|}
\hline \multirow[b]{2}{*}{$\mathrm{m}$} & \multicolumn{3}{|c|}{ Only scale uncertainty } & \multicolumn{3}{|c|}{ Only PDF uncertainty } & \multicolumn{3}{|c|}{ Total uncertainty } \\
\hline & $\min$ & $\max$ & $\delta[\%]$ & $\min$ & $\max$ & $\delta[\%]$ & $\min$ & $\max$ & $\delta[\%]$ \\
\hline 165 & 9.26 & 9.66 & 3 & 9.04 & 10.1 & 6 & 8.73 & 10.1 & 8 \\
\hline 166 & 8.97 & 9.36 & 3 & 8.76 & 9.82 & 6 & 8.46 & 9.87 & 8 \\
\hline 167 & 8.68 & 9.07 & 3 & 8.49 & 9.51 & 6 & 8.2 & 9.57 & 8 \\
\hline 168 & 8.41 & 8.79 & 3 & 8.22 & 9.22 & 6 & 7.94 & 9.27 & 8 \\
\hline 169 & 8.15 & 8.52 & 3 & 7.97 & 8.93 & 6 & 7.7 & 8.98 & 8 \\
\hline 170 & 7.9 & 8.26 & 3 & 7.73 & 8.65 & 6 & 7.46 & 8.7 & 8 \\
\hline 171 & 7.65 & 8.01 & 3 & 7.49 & 8.38 & 6 & 7.23 & 8.44 & 8 \\
\hline 172 & 7.42 & 7.76 & 3 & 7.26 & 8.12 & 6 & 7.01 & 8.18 & 8 \\
\hline 173 & 7.19 & 7.53 & 3 & 7.04 & 7.87 & 6 & 6.8 & 7.93 & 8 \\
\hline 174 & 6.97 & 7.3 & 3 & 6.83 & 7.63 & 6 & 6.59 & 7.69 & 8 \\
\hline 175 & 6.76 & 7.08 & 3 & 6.62 & 7.4 & 6 & 6.39 & 7.45 & 8 \\
\hline 176 & 6.55 & 6.87 & 3 & 6.43 & 7.17 & 6 & 6.2 & 7.23 & 8 \\
\hline 177 & 6.36 & 6.66 & 3 & 6.23 & 6.96 & 6 & 6.01 & 7.01 & 8 \\
\hline 178 & 6.16 & 6.46 & 3 & 6.05 & 6.75 & 6 & 5.83 & 6.8 & 8 \\
\hline 179 & 5.98 & 6.27 & 3 & 5.87 & 6.54 & 6 & 5.66 & 6.6 & 8 \\
\hline 180 & 5.8 & 6.08 & 3 & 5.69 & 6.35 & 6 & 5.49 & 6.4 & 8 \\
\hline
\end{tabular}

TABLE VII. Same as in Table VI using the MRST-2006 NNLO PDF set [25].

\begin{tabular}{|c|c|c|c|c|c|c|c|c|c|}
\hline \multirow[b]{2}{*}{$\mathrm{m}$} & \multicolumn{3}{|c|}{ Only scale uncertainty } & \multicolumn{3}{|c|}{ Only PDF uncertainty } & \multicolumn{3}{|c|}{ Total uncertainty } \\
\hline & $\min$ & $\max$ & $\delta[\%]$ & $\min$ & $\max$ & $\delta[\%]$ & $\min$ & $\max$ & $\delta[\%]$ \\
\hline 165 & 9.59 & 10 & 3 & 9.73 & 10.2 & 3 & 9.34 & 10.3 & 6 \\
\hline 166 & 9.28 & 9.76 & 3 & 9.42 & 9.94 & 3 & 9.04 & 10 & 6 \\
\hline 167 & 8.99 & 9.45 & 3 & 9.12 & 9.62 & 3 & 8.75 & 9.7 & 6 \\
\hline 168 & 8.7 & 9.16 & 3 & 8.83 & 9.31 & 3 & 8.47 & 9.39 & 6 \\
\hline 169 & 8.42 & 8.87 & 3 & 8.55 & 9.02 & 3 & 8.2 & 9.1 & 6 \\
\hline 170 & 8.16 & 8.59 & 3 & 8.28 & 8.73 & 3 & 7.94 & 8.81 & 6 \\
\hline 171 & 7.9 & 8.32 & 3 & 8.02 & 8.46 & 3 & 7.69 & 8.53 & 6 \\
\hline 172 & 7.65 & 8.06 & 3 & 7.77 & 8.19 & 3 & 7.45 & 8.27 & 6 \\
\hline 173 & 7.41 & 7.81 & 3 & 7.53 & 7.93 & 3 & 7.22 & 8.01 & 6 \\
\hline 174 & 7.18 & 7.57 & 3 & 7.29 & 7.69 & 3 & 6.99 & 7.76 & 6 \\
\hline 175 & 6.95 & 7.34 & 3 & 7.07 & 7.45 & 3 & 6.77 & 7.52 & 6 \\
\hline 176 & 6.74 & 7.11 & 3 & 6.85 & 7.22 & 3 & 6.57 & 7.29 & 6 \\
\hline 177 & 6.53 & 6.89 & 3 & 6.64 & 6.99 & 3 & 6.36 & 7.07 & 6 \\
\hline 178 & 6.33 & 6.68 & 3 & 6.43 & 6.78 & 3 & 6.17 & 6.85 & 6 \\
\hline 179 & 6.13 & 6.48 & 3 & 6.24 & 6.57 & 3 & 5.98 & 6.64 & 6 \\
\hline 180 & 5.95 & 6.28 & 3 & 6.05 & 6.37 & 3 & 5.8 & 6.44 & 6 \\
\hline
\end{tabular}



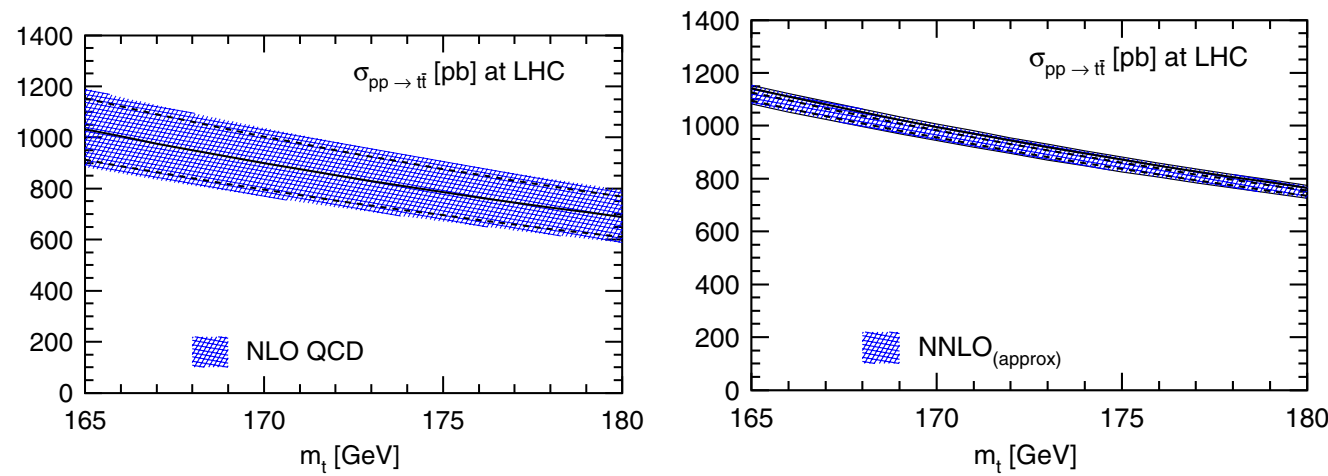

FIG. 9 (color online). The NNLO (approximate) QCD prediction for the $t \bar{t}$ total cross section at LHC as functions of $m_{t}$ for $\sqrt{s_{\text {had }}}=$ $14 \mathrm{TeV}$ (right). The solid line is the central value for $\mu=m_{t}$, the dashed lower and upper lines correspond to $\mu=2 m_{t}$ and $\mu=m_{t} / 2$, respectively. The band denotes the total uncertainty that is the uncertainty due to scale variations and the PDF uncertainty of the MRST-2006 NNLO set [25]. For comparison, the left plot shows the corresponding prediction at NLO accuracy using the PDF set CTEQ6.5 [24].

minor discrepancy might be attributed to slightly different input densities at low scale. Compared to the NLO results the scale dependence of $\sigma_{\mathrm{NNLO}(\text { approx) }}$ is improved. In particular, we find a plateau at $\mu=m_{t}$. Note that due to the different shape of the NNLO curve the uncertainty estimate of Eq. (4) has to be adapted here. Restricting the scale to the interval $\left[m_{t} / 2,2 m_{t}\right]$ the residual scale dependence of $\sigma_{\mathrm{NNLO}(\text { approx) }}$ is reduced to a few percent.

In Fig. 8 we plot the total cross section at Tevatron and display also CDF data [41] with $m_{t}=171 \mathrm{GeV}$. The corresponding cross section values are given in Tables VI and VII. By using $\sigma_{\mathrm{NNLO}}$ (approx) the residual scale dependence is reduced to $3 \%$. Compared to $\sigma_{\mathrm{NLO}}$ and $\sigma_{\text {res }}$ presented in the previous section, this corresponds to a reduction by a factor of 2 . The data points nicely agree with the theoretical prediction. The overall uncertainty of the theoretical prediction is about $8 \%$ for CTEQ6.5 and 6\% for MRST-2006 NNLO-an accuracy unlikely to be reached at the Tevatron experiments. Note that the smaller PDF uncertainty obtained when using the MRST-2006 NNLO PDF set is due to a different convention used by the MRST collaboration to define the PDF uncertainty. In Fig. 9 (right) we show $\sigma_{\mathrm{NNLO}}$ (approx) for the LHC. Again we observe a drastic reduction of the scale uncertainty compared to $\sigma_{\mathrm{NLO}}$ and $\sigma_{\text {res }}$. For comparison we show also $\sigma_{\mathrm{NLO}}$ in Fig. 9 (left). The corresponding cross section values are listed in Tables VIII and IX. Apart from reducing the scale dependence, the $\sigma_{\mathrm{NNLO}(\text { approx) }}$ leads only to a small shift of a few percent in the central value for the cross section prediction. The $\sigma_{\mathrm{NNLO}(\text { approx) }}$ band is well con-

TABLE VIII. The cross section $\sigma_{\text {NNLO(approx) }}$ as derived in this paper in pb for various values of the top-quark mass $m_{t}$ at LHC $\left(\sqrt{s_{\text {had }}}=14 \mathrm{TeV}\right)$ using the CTEQ6.5 PDF set [24].

\begin{tabular}{|c|c|c|c|c|c|c|c|c|c|}
\hline \multirow[b]{2}{*}{$\underline{\mathrm{m}}$} & \multicolumn{3}{|c|}{ Only scale uncertainty } & \multicolumn{3}{|c|}{ Only PDF uncertainty } & \multicolumn{3}{|c|}{ Total uncertainty } \\
\hline & $\min$ & $\max$ & $\delta[\%]$ & $\min$ & $\max$ & $\delta[\%]$ & $\min$ & $\max$ & $\delta[\%]$ \\
\hline 165 & 1035 & 1082 & 3 & 1048 & 1117 & 4 & 1003 & 1117 & 6 \\
\hline 166 & 1007 & 1052 & 3 & 1019 & 1086 & 4 & 975 & 1086 & 6 \\
\hline 167 & 979 & 1024 & 3 & 991 & 1056 & 4 & 948 & 1056 & 6 \\
\hline 168 & 953 & 996 & 3 & 964 & 1028 & 4 & 922 & 1028 & 6 \\
\hline 169 & 927 & 969 & 3 & 937 & 1000 & 4 & 897 & 1000 & 6 \\
\hline 170 & 902 & 943 & 3 & 912 & 973 & 4 & 873 & 973 & 6 \\
\hline 171 & 878 & 917 & 3 & 887 & 947 & 4 & 849 & 947 & 6 \\
\hline 172 & 855 & 893 & 3 & 863 & 922 & 4 & 827 & 922 & 6 \\
\hline 173 & 832 & 869 & 3 & 840 & 898 & 4 & 805 & 898 & 6 \\
\hline 174 & 810 & 846 & 3 & 818 & 874 & 4 & 783 & 874 & 6 \\
\hline 175 & 789 & 824 & 3 & 796 & 851 & 4 & 762 & 851 & 6 \\
\hline 176 & 768 & 802 & 3 & 775 & 829 & 4 & 742 & 829 & 6 \\
\hline 177 & 748 & 781 & 3 & 755 & 808 & 4 & 723 & 808 & 6 \\
\hline 178 & 729 & 761 & 3 & 735 & 787 & 4 & 704 & 787 & 6 \\
\hline 179 & 710 & 741 & 3 & 716 & 767 & 4 & 686 & 767 & 6 \\
\hline 180 & 692 & 722 & 3 & 698 & 747 & 4 & 668 & 747 & 6 \\
\hline
\end{tabular}


TABLE IX. Same as in Table VIII using the MRST-2006 NNLO PDF set [25].

\begin{tabular}{|c|c|c|c|c|c|c|c|c|c|}
\hline \multirow[b]{2}{*}{$\mathrm{m}$} & \multicolumn{3}{|c|}{ Only scale uncertainty } & \multicolumn{3}{|c|}{ Only PDF uncertainty } & \multicolumn{3}{|c|}{ Total uncertainty } \\
\hline & $\min$ & $\max$ & $\delta[\%]$ & $\min$ & $\max$ & $\delta[\%]$ & $\min$ & $\max$ & $\delta[\%]$ \\
\hline 165 & 1094 & 1141 & 3 & 1128 & 1154 & 2 & 1082 & 1154 & 4 \\
\hline 166 & 1064 & 1110 & 3 & 1097 & 1122 & 2 & 1052 & 1122 & 4 \\
\hline 167 & 1035 & 1080 & 3 & 1067 & 1092 & 2 & 1024 & 1092 & 4 \\
\hline 168 & 1008 & 1050 & 3 & 1038 & 1063 & 2 & 996 & 1063 & 4 \\
\hline 169 & 981 & 1022 & 3 & 1010 & 1034 & 2 & 969 & 1034 & 4 \\
\hline 170 & 955 & 995 & 3 & 983 & 1007 & 2 & 943 & 1007 & 4 \\
\hline 171 & 929 & 969 & 3 & 957 & 980 & 2 & 918 & 980 & 4 \\
\hline 172 & 905 & 943 & 3 & 932 & 954 & 2 & 894 & 954 & 4 \\
\hline 173 & 881 & 918 & 3 & 907 & 929 & 2 & 871 & 929 & 4 \\
\hline 174 & 858 & 894 & 3 & 883 & 905 & 2 & 848 & 905 & 4 \\
\hline 175 & 836 & 871 & 3 & 860 & 882 & 2 & 826 & 882 & 4 \\
\hline 176 & 814 & 848 & 3 & 838 & 859 & 2 & 804 & 859 & 4 \\
\hline 177 & 793 & 826 & 3 & 816 & 837 & 2 & 783 & 837 & 4 \\
\hline 178 & 773 & 805 & 3 & 795 & 815 & 2 & 763 & 815 & 4 \\
\hline 179 & 753 & 785 & 3 & 775 & 795 & 2 & 744 & 795 & 4 \\
\hline 180 & 734 & 765 & 3 & 755 & 774 & 2 & 725 & 774 & 4 \\
\hline
\end{tabular}

tained in the $\sigma_{\mathrm{NLO}}$ band. So perturbation theory seems to be well behaved and under control. The overall uncertainty is about $6 \%$ for the CTEQ6.5 PDF set and about $4 \%$ for the MRST-2006 NNLO set.

The numbers quoted in Tables VI, VII, VIII, and IX represent presently the best estimates for the top-quark production cross section at Tevatron and LHC (see Appendix B for additional information on the individual PDFs and their eigenvalues). It should be kept in mind, though, that there is an intrinsic uncertainty in the central value at $\mu=m_{t}$ of our NNLO (approximate) result due to neglected power corrections in $\beta \sim(1-\rho)$ away from threshold. However, due to the steeply falling parton flux (see Figs. 1 and 2), the numerical impact of these contributions is much suppressed.

\section{CONCLUSIONS}

In this article we have summarized the present knowledge on theory predictions for the top-quark pair production cross section at Tevatron and LHC. We have taken some care to quantify the sensitivity of the total cross section to soft-gluon emission and large Sudakov-type logarithms. As is well known, top-quark pair production at Tevatron is largely dominated by parton kinematics close to threshold, thus approximations based on softgluon resummation should provide an excellent description. At LHC we find that soft-gluon emission near threshold is less dominant, but contributes still a numerically sizable fraction to the total cross section. Thus, soft-gluon effects in $t \bar{t}$-production are still rather prominent at LHC as well.

We have updated the NLL resummed cross section as defined in Refs. $[8,14]$ using recent PDFs. Furthermore, we have extended the resummed predictions to NNLL accu- racy and we have derived approximate NNLO cross sections which are exact to all powers in $\ln \beta$ at two loops. Together with the exact NNLO scale dependence (and including the two-loop Coulomb corrections) our result for $\sigma_{\mathrm{NNLO} \text { (approx) }}$ represents the best present estimate for hadroproduction of top-quark pairs, both at Tevatron and LHC. As mentioned earlier we believe that hard corrections at the NNLO level are small. This is supported by the explicit findings of Ref. [15]. We have found for the NNLL resummed cross section and the finite-order expansion good apparent convergence properties. Moreover, the stability of the total cross section with respect to scale variations is much improved by our NNLO (approximate) result.

In closing let us briefly comment on ideas to use topquark pair production as an additional calibration process for the parton luminosity at LHC [11]. This could become feasible because the PDF dependence of $t \bar{t}$ production at LHC is anticorrelated with $W / Z$-boson production (the standard candle process at LHC, see e.g. [42,43]) and correlated with Higgs boson production, especially for larger Higgs masses. It has been noted, however, that the NLO theory predictions to the top-quark cross section are not accurate enough. We are confident that the NNLO (approximate) results of this present paper provide a step in the right direction by further constraining the theory uncertainties for this important process.

The complete NNLO QCD predictions for heavy-quark hadroproduction do not only require the hard scattering cross section but also the evolution of the parton densities to be performed at the same order employing the NNLO splitting functions [29,30]. In addition, we remark that the present accuracy on the gluon PDF in the medium $x$ range of interest for top-quark production at LHC is well con- 
strained from DIS data for structure functions in $e^{ \pm} p$ scattering from HERA and evolution, leading to the rather small uncertainty of 3\% (see Fig. 1) at small energies. Thus, for top-quark pair production to become a standard candle process at LHC similar to $W / Z$ gauge boson production and to become competitive with DIS data, also an experimental accuracy much better than the currently quoted [1] value of $10 \%$ will be needed.

\section{ACKNOWLEDGMENTS}

We would like to thank S. Alekhin, W. Bernreuther, and E. Laenen for stimulating discussions. S. M. is supported by the Helmholtz Gemeinschaft under Contract No. VHNG-105. P. U. is supported by DFG. This work is also partly supported by DFG in SFB/TR 9 and the hospitality of the Galileo-Galilei Institute in Florence is gratefully acknowledged. We would also like to thank J. Kühn for pointing out an error in the Coulomb contributions at two loops in Eqs. (19), (21), (A18), and (A20), respectively, in the first version of this article.

Note added.-Shortly after submitting the preprint version of this article, the related work [44] appeared, where a similar study has been performed. In the meantime a tuned comparison of the resummed (NLL) partonic cross section has been done and we have found good agreement, once it was taken into account that the matching for the scheme $A=2$ implemented in [44] is slightly different from the original Ref. [8] employed in the present article. This minor scheme dependence accounts for the observed differences in the predictions for the total cross section between [44] and this article.

\section{APPENDIX A: USEFUL FORMULAS}

The Born cross section in the color basis defined by color-singlet and color-octet final states reads in momentum space with $\rho=4 m_{t}^{2} / s$ and $\beta=\sqrt{1-\rho}$ :

$\hat{\sigma}_{q \bar{q}, 1}^{(0)}(\rho)=0, \quad \hat{\sigma}_{q \bar{q}, 8}^{(0)}(\rho)=\frac{\alpha_{s}^{2}}{m_{t}^{2}} \frac{\pi}{12} \frac{C_{F}}{C_{A}} \beta \rho(2+\rho)$,

$\hat{\sigma}_{g g, 1}^{(0)}(\rho)=\frac{\alpha_{s}^{2}}{m_{t}^{2}} \frac{\pi}{16} \frac{1}{C_{A}\left(C_{A}^{2}-1\right)} \beta \rho\left\{\left(4+4 \rho-2 \rho^{2}\right) \frac{1}{\beta} \ln \frac{1+\beta}{1-\beta}-4-4 \rho\right\}$,

$\hat{\sigma}_{g g, 8}^{(0)}(\rho)=\frac{\alpha_{s}^{2}}{m_{t}^{2}} \frac{\pi}{24} \frac{1}{C_{A}^{2}-1} \beta \rho\left\{12 C_{F}\left[\left(2+2 \rho-\rho^{2}\right) \frac{1}{\beta} \ln \frac{1+\beta}{1-\beta}-2-2 \rho\right]-C_{A}\left[6\left(1+\rho-\rho^{2}\right) \frac{1}{\beta} \ln \frac{1+\beta}{1-\beta}-2-\rho\right]\right\}$,

where $C_{A}$ and $C_{F}$ are the usual color factors, with $C_{A}=$ $N=3$ and $C_{F}=\frac{1}{2 N}\left(N^{2}-1\right)=4 / 3$ in QCD. The Mellin moments as defined in Eq. (7) can be easily computed, see e.g. Ref. [8].

Next we present the perturbative expansions for the anomalous dimensions $A_{q}, A_{g}, D_{q}$, and $D_{g}$ entering Eq. (13). We have for the quark case $[29,37]$

$$
\begin{aligned}
A_{q}^{(1)}= & 4 C_{F}, \quad A_{q}^{(2)}=8 C_{F}\left[\left(\frac{67}{18}-\zeta_{2}\right) C_{A}-\frac{5}{9} n_{f}\right] \\
A_{q}^{(3)}= & 16 C_{F}\left[C_{A}^{2}\left(\frac{245}{24}-\frac{67}{9} \zeta_{2}+\frac{11}{6} \zeta_{3}+\frac{11}{5} \zeta_{2}^{2}\right)\right. \\
& +C_{F} n_{f}\left(-\frac{55}{24}+2 \zeta_{3}\right)+C_{A} n_{f}\left(-\frac{209}{108}\right. \\
& \left.\left.+\frac{10}{9} \zeta_{2}-\frac{7}{3} \zeta_{3}\right)+n_{f}^{2}\left(-\frac{1}{27}\right)\right],
\end{aligned}
$$

where $n_{f}$ denotes the number of effectively massless quark flavors and $\zeta_{2}, \zeta_{3}, \ldots$ are the values of the Riemann zeta function. Likewise, the $D_{q}$ read

$$
\begin{aligned}
D_{q}^{(1)}= & 0, \\
D_{q}^{(2)}= & C_{F}\left[C_{A}\left(-\frac{1616}{27}+\frac{176}{3} \zeta_{2}+56 \zeta_{3}\right)\right. \\
& \left.+n_{f}\left(\frac{224}{27}-\frac{32}{3} \zeta_{2}\right)\right] .
\end{aligned}
$$

All gluonic quantities are given by the simple relation

$$
A_{g}^{(i)}=\frac{C_{A}}{C_{F}} A_{q}^{(i)}, \quad D_{g}^{(i)}=\frac{C_{A}}{C_{F}} D_{q}^{(i)}
$$

Here we summarize the functions $g_{i j}^{1}, g_{i j, I}^{2}, g_{i j, I}^{3}$ appearing in the resummed cross section Eq. (10) to NNLL accuracy [26-28]. Keeping the full dependence on $\mu_{r}$ and $\mu_{f}$, we have

$$
g_{q \bar{q}}^{1}=A_{q}^{(1)}\left(2-2 \ln (1-2 \lambda)+\lambda^{-1} \ln (1-2 \lambda)\right)
$$

$$
\begin{aligned}
g_{q \bar{q}, 1}^{2}= & \left(A_{q}^{(1)} \beta_{1}-A_{q}^{(2)}\right)(2 \lambda+\ln (1-2 \lambda)) \\
& +\frac{1}{2} A_{q}^{(1)} \beta_{1} \ln ^{2}(1-2 \lambda)-\frac{1}{2}\left(4 A_{q}^{(1)} \gamma_{e}-D_{q}^{(1)}\right) \\
& \times \ln (1-2 \lambda)+\ln \left(4 m^{2} / \mu_{r}^{2}\right) A_{q}^{(1)} \ln (1-2 \lambda) \\
& +2 \ln \left(\mu_{f}^{2} / \mu_{r}^{2}\right) A_{q}^{(1)} \lambda, \\
& g_{q \bar{q}, 8}^{2}=g_{q \bar{q}, 1}^{2}-\frac{1}{2} \ln (1-2 \lambda) D_{Q \bar{Q}}^{(1)},
\end{aligned}
$$




$$
\begin{aligned}
g_{q \bar{q}, 1}^{3}= & \frac{1}{2}\left(A_{q}^{(1)} \beta_{2}-A_{q}^{(1)} \beta_{1}^{2}+A_{q}^{(2)} \beta_{1}-A_{q}^{(3)}\right)\left(1+2 \lambda-\frac{1}{1-2 \lambda}\right)+A_{q}^{(1)} \beta_{1}^{2}\left(\frac{\ln (1-2 \lambda)}{1-2 \lambda}+\frac{1}{2} \frac{\ln ^{2}(1-2 \lambda)}{1-2 \lambda}\right) \\
& +\left(A_{q}^{(1)} \beta_{2}-A_{q}^{(1)} \beta_{1}^{2}\right) \ln (1-2 \lambda)+\left(2 A_{q}^{(1)} \beta_{1} \gamma_{e}+A_{q}^{(2)} \beta_{1}-\frac{1}{2} D_{q}^{(1)} \beta_{1}\right)\left(1-\frac{1}{1-2 \lambda}-\frac{\ln (1-2 \lambda)}{1-2 \lambda}\right) \\
& -\left(A_{q}^{(1)} \beta_{2}+2 A_{q}^{(1)}\left(\gamma_{e}^{2}+\zeta_{2}\right)+2 A_{q}^{(2)} \gamma_{e}-D_{q}^{(1)} \gamma_{e}-\frac{1}{2} D_{q}^{(2)}\right)\left(1-\frac{1}{1-2 \lambda}\right) \\
& +\ln \left(4 m^{2} / \mu_{r}^{2}\right)\left[\left(2 A_{q}^{(1)} \gamma_{e}-A_{q}^{(1)} \beta_{1}+A_{q}^{(2)}-\frac{1}{2} D_{q}^{(1)}\right)\left(1-\frac{1}{1-2 \lambda}\right)+A_{q}^{(1)} \beta_{1}\left(\frac{\ln (1-2 \lambda)}{1-2 \lambda}\right)\right] \\
& +2 \ln \left(\mu_{f}^{2} / \mu_{r}^{2}\right) A_{q}^{(2)} \lambda-\frac{1}{2} \ln ^{2}\left(4 m^{2} / \mu_{r}^{2}\right) A_{q}^{(1)}\left(1-\frac{1}{1-2 \lambda}\right)-\ln ^{2}\left(\mu_{f}^{2} / \mu_{r}^{2}\right) A_{q}^{(1)} \lambda, \\
& g_{q \bar{q}, 8}^{3}=g_{q \bar{q}, 1}^{3}-\frac{1}{2}\left(D_{Q \bar{Q}}^{(2)}+2 D_{Q \bar{Q}}^{(1)} \gamma_{e}-D_{Q \bar{Q}}^{(1)} \beta_{1}\right)\left(1-\frac{1}{(1-2 \lambda)}\right)-\frac{1}{2} \frac{\ln (1-2 \lambda)}{(1-2 \lambda)} D_{Q \bar{Q}}^{(1)} \beta_{1} \\
& +\frac{1}{2} \ln \left(4 m^{2} / \mu_{r}^{2}\right) D_{Q \bar{Q}}^{(1)}\left(1-\frac{1}{(1-2 \lambda)}\right),
\end{aligned}
$$

with $\gamma_{e}=0.5772167$. The gluonic expressions $g_{g g}^{1}, g_{g g, I}^{2}$, and $g_{g g, I}^{3}$ are obtained with the obvious replacements $A_{q}^{(i)} \rightarrow A_{g}^{(i)}$ and $D_{q}^{(i)} \rightarrow D_{g}^{(i)}$. The dependence on $\beta_{0}$ is recovered by $A^{(i)} \rightarrow A^{(i)} / \beta_{0}^{i}, D^{(i)} \rightarrow D^{(i)} / \beta_{0}^{i}, \beta_{i} \rightarrow \beta_{i} / \beta_{0}^{i+1}$ and multiplication of $g_{i j, I}^{3}$ by $\beta_{0}$. We also give explicit results for the matching functions $g_{i j, I}^{0}$ in Eq. (9),

$$
g_{q \bar{q}, 1}^{0}=0,
$$

$$
\begin{aligned}
& g_{q \bar{q}, 8}^{0}=1+a_{s}\left\{C_{F}\left[-64+8 \gamma_{e}^{2}+4 \pi^{2}-32 \ln 2+8 \ln ^{2} 2\right]+C_{A}\left[-8+4 \gamma_{e}-4 \ln 2\right]+4 C_{q \bar{q}}^{(1)}+\ln \left(4 m^{2} / \mu_{r}^{2}\right) C_{F}\left[16-8 \gamma_{e}\right]\right\} \\
& +a_{s}^{2}\left\{C_{F} C_{q \bar{q}}^{(1)}\left[-256+32 \gamma_{e}^{2}+16 \pi^{2}+384 \ln 2-64 \gamma_{e} \ln 2-256 \ln ^{2} 2\right]+C_{F} n_{f}\left[\frac{6976}{27}-\frac{448}{9} \zeta_{3}-\frac{224}{27} \gamma_{e}\right.\right. \\
& \left.-\frac{80}{9} \gamma_{e}^{2}-\frac{32}{9} \gamma_{e}^{3}-\frac{136}{9} \pi^{2}-\frac{10240}{27} \ln 2+\frac{160}{9} \gamma_{e} \ln 2+\frac{32}{3} \gamma_{e}^{2} \ln 2+16 \pi^{2} \ln 2+\frac{2368}{9} \ln ^{2} 2-\frac{32}{3} \gamma_{e} \ln ^{2} 2-\frac{832}{9} \ln ^{3} 2\right] \\
& +C_{F}^{2}\left[-8192+3584 \zeta_{3}-512 \gamma_{e}^{2}+32 \gamma_{e}^{4}+256 \pi^{2}+32 \pi^{2} \gamma_{e}^{2}+24 \pi^{4}+12288 \ln 2-5376 \zeta_{3} \ln 2+1024 \gamma_{e} \ln 2\right. \\
& \left.-128 \gamma_{e}^{3} \ln 2-768 \pi^{2} \ln 2-64 \pi^{2} \gamma_{e} \ln 2-9728 \ln ^{2} 2+192 \gamma_{e}^{2} \ln ^{2} 2+608 \pi^{2} \ln ^{2} 2+6912 \ln ^{3} 2-128 \gamma_{e} \ln ^{3} 2-2560 \ln ^{4} 2\right] \\
& +C_{A} C_{q \bar{q}}^{(1)}\left[-32+16 \gamma_{e}+32 \ln 2\right]+C_{A} n_{f}\left[\frac{272}{9}-\frac{40}{9} \gamma_{e}-\frac{8}{3} \gamma_{e}^{2}-\frac{4}{3} \pi^{2}-\frac{368}{9} \ln 2+\frac{16}{3} \gamma_{e} \ln 2+\frac{64}{3} \ln ^{2} 2\right] \\
& +C_{A} C_{F}\left[-\frac{55264}{27}+\frac{7504}{9} \zeta_{3}-\frac{5296}{27} \gamma_{e}-56 \gamma_{e} \zeta_{3}-\frac{40}{9} \gamma_{e}^{2}+\frac{464}{9} \gamma_{e}^{3}+\frac{1276}{9} \pi^{2}+16 \pi^{2} \gamma_{e}-\frac{8}{3} \pi^{2} \gamma_{e}^{2}-\frac{4}{3} \pi^{4}\right. \\
& +\frac{88192}{27} \ln 2-112 \zeta_{3} \ln 2+\frac{80}{9} \gamma_{e} \ln 2-\frac{464}{3} \gamma_{e}^{2} \ln 2-232 \pi^{2} \ln 2+\frac{16}{3} \pi^{2} \gamma_{e} \ln 2-\frac{24736}{9} \ln ^{2} 2+\frac{464}{3} \gamma_{e} \ln ^{2} 2 \\
& \left.+\frac{64}{3} \pi^{2} \ln ^{2} 2+\frac{12064}{9} \ln ^{3} 2\right]+C_{A}^{2}\left[-\frac{1592}{9}-\frac{20}{9} \gamma_{e}+\frac{68}{3} \gamma_{e}^{2}+14 \pi^{2}-\frac{4}{3} \pi^{2} \gamma_{e}+\frac{2408}{9} \ln 2-\frac{136}{3} \gamma_{e} \ln 2-\frac{8}{3} \pi^{2} \ln 2\right. \\
& \left.-\frac{544}{3} \ln ^{2} 2\right]+C_{q \bar{q}}^{(2)}+\ln \left(\mu_{f}^{2} / \mu_{r}^{2}\right) C_{F} C_{q \bar{q}}^{(1)}\left[64-32 \gamma_{e}-64 \ln 2\right]+\ln \left(\mu_{f}^{2} / \mu_{r}^{2}\right) C_{F} n_{f}\left[-\frac{544}{9}+\frac{80}{9} \gamma_{e}+\frac{16}{3} \gamma_{e}^{2}+\frac{8}{3} \pi^{2}\right. \\
& \left.+\frac{736}{9} \ln 2-\frac{32}{3} \gamma_{e} \ln 2-\frac{128}{3} \ln ^{2} 2\right]+\ln \left(\mu_{f}^{2} / \mu_{r}^{2}\right) C_{F}^{2}\left[1024-896 \zeta_{3}+512 \gamma_{e}+128 \gamma_{e}^{2}-64 \gamma_{e}^{3}-64 \pi^{2}-32 \pi^{2} \gamma_{e}\right. \\
& \left.-1536 \ln 2-256 \gamma_{e} \ln 2+128 \gamma_{e}^{2} \ln 2+192 \pi^{2} \ln 2+1664 \ln ^{2} 2-64 \gamma_{e} \ln ^{2} 2-1152 \ln ^{3} 2\right]+\ln \left(\mu_{f}^{2} / \mu_{r}^{2}\right) C_{A} n_{f} \\
& \times\left[-\frac{16}{3}+\frac{8}{3} \gamma_{e}+\frac{16}{3} \ln 2\right]+\ln \left(\mu_{f}^{2} / \mu_{r}^{2}\right) C_{A} C_{F}\left[\frac{3184}{9}+\frac{616}{9} \gamma_{e}-\frac{184}{3} \gamma_{e}^{2}-36 \pi^{2}+\frac{8}{3} \pi^{2} \gamma_{e}-\frac{4816}{9} \ln 2+\frac{272}{3} \gamma_{e} \ln 2\right. \\
& \left.+\frac{16}{3} \pi^{2} \ln 2+\frac{1280}{3} \ln ^{2} 2\right]+\ln \left(\mu_{f}^{2} / \mu_{r}^{2}\right) C_{A}^{2}\left[\frac{88}{3}-\frac{44}{3} \gamma_{e}-\frac{88}{3} \ln 2\right]+\ln ^{2}\left(\mu_{f}^{2} / \mu_{r}^{2}\right) C_{F} n_{f}\left[\frac{16}{3}-\frac{8}{3} \gamma_{e}-\frac{16}{3} \ln 2\right] \\
& \left.+\ln ^{2}\left(\mu_{f}^{2} / \mu_{r}^{2}\right) C_{F}^{2}\left[-128 \gamma_{e}+32 \gamma_{e}^{2}+16 \pi^{2}-128 \ln ^{2} 2\right]+\ln ^{2}\left(\mu_{f}^{2} / \mu_{r}^{2}\right) C_{A} C_{F}\left[-\frac{88}{3}+\frac{44}{3} \gamma_{e}+\frac{88}{3} \ln 2\right]\right\},
\end{aligned}
$$




$$
\begin{aligned}
& g_{g g, 1}^{0}=1+a_{s}\left\{C_{A}\left[-64+8 \gamma_{e}^{2}+4 \pi^{2}-32 \ln 2+8 \ln ^{2} 2\right]+4 C_{g g}^{(1)}+\ln \left(4 m^{2} / \mu_{r}^{2}\right) C_{A}\left[16-8 \gamma_{e}\right]\right\}+a_{s}^{2}\left\{C_{A} C_{g g}^{(1)}[-256\right. \\
& \left.+32 \gamma_{e}^{2}+16 \pi^{2}+384 \ln 2-64 \gamma_{e} \ln 2-256 \ln ^{2} 2\right]+C_{A} n_{f}\left[\frac{6976}{27}-\frac{448}{9} \zeta_{3}-\frac{224}{27} \gamma_{e}-\frac{80}{9} \gamma_{e}^{2}-\frac{32}{9} \gamma_{e}^{3}-\frac{136}{9} \pi^{2}\right. \\
& \left.-\frac{10240}{27} \ln 2+\frac{160}{9} \gamma_{e} \ln 2+\frac{32}{3} \gamma_{e}^{2} \ln 2+16 \pi^{2} \ln 2+\frac{2368}{9} \ln ^{2} 2-\frac{32}{3} \gamma_{e} \ln ^{2} 2-\frac{832}{9} \ln ^{3} 2\right]+C_{A}^{2}\left[-\frac{262624}{27}\right. \\
& +\frac{35728}{9} \zeta_{3}+\frac{1616}{27} \gamma_{e}-56 \gamma_{e} \zeta_{3}-\frac{4072}{9} \gamma_{e}^{2}+\frac{176}{9} \gamma_{e}^{3}+32 \gamma_{e}^{4}+\frac{3292}{9} \pi^{2}+\frac{88}{3} \pi^{2} \gamma_{e}^{2}+\frac{68}{3} \pi^{4}+\frac{392320}{27} \ln 2 \\
& -5488 \zeta_{3} \ln 2+\frac{8144}{9} \gamma_{e} \ln 2-\frac{176}{3} \gamma_{e}^{2} \ln 2-128 \gamma_{e}^{3} \ln 2-888 \pi^{2} \ln 2-\frac{176}{3} \pi^{2} \gamma_{e} \ln 2-\frac{101344}{9} \ln ^{2} 2+\frac{176}{3} \gamma_{e} \ln ^{2} 2 \\
& \left.+192 \ln ^{2} 2 \gamma_{e}^{2}+\frac{1888}{3} \pi^{2} \ln ^{2} 2+\frac{66784}{9} \ln ^{3} 2-128 \gamma_{e} \ln ^{3} 2-2560 \ln ^{4} 2\right]+C_{g g}^{(2)}+\ln \left(\mu_{f}^{2} / \mu_{r}^{2}\right) C_{A} C_{g g}^{(1)}\left[64-32 \gamma_{e}\right. \\
& -64 \ln 2]+\ln \left(\mu_{f}^{2} / \mu_{r}^{2}\right) C_{A} n_{f}\left[-\frac{544}{9}+\frac{80}{9} \gamma_{e}+\frac{16}{3} \gamma_{e}^{2}+\frac{8}{3} \pi^{2}+\frac{736}{9} \ln 2-\frac{32}{3} \gamma_{e} \ln 2-\frac{128}{3} \ln ^{2} 2\right] \\
& +\ln \left(\mu_{f}^{2} / \mu_{r}^{2}\right) C_{A}^{2}\left[\frac{12400}{9}-896 \zeta_{3}+\frac{4072}{9} \gamma_{e}+\frac{296}{3} \gamma_{e}^{2}-64 \gamma_{e}^{3}-84 \pi^{2}-\frac{88}{3} \pi^{2} \gamma_{e}-\frac{18064}{9} \ln 2-\frac{592}{3} \gamma_{e} \ln 2\right. \\
& \left.+128 \gamma_{e}^{2} \ln 2+\frac{592}{3} \pi^{2} \ln 2+\frac{5696}{3} \ln ^{2} 2-64 \gamma_{e} \ln ^{2} 2-1152 \ln ^{3} 2\right]+\ln ^{2}\left(\mu_{f}^{2} / \mu_{r}^{2}\right) C_{A} n_{f}\left[\frac{16}{3}-\frac{8}{3} \gamma_{e}-\frac{16}{3} \ln 2\right] \\
& \left.+\ln ^{2}\left(\mu_{f}^{2} / \mu_{r}^{2}\right) C_{A}^{2}\left[-\frac{88}{3}-\frac{340}{3} \gamma_{e}+32 \gamma_{e}^{2}+16 \pi^{2}+\frac{88}{3} \ln 2-128 \ln ^{2} 2\right]\right\}, \\
& g_{g g, 8}^{0}=g_{g g, 1}^{0}+a_{s}\left\{C_{A}\left[-8+4 \gamma_{e}-4 \ln 2\right]\right\}+a_{s}^{2}\left\{C_{A} C_{g g}^{(1)}\left[-32+16 \gamma_{e}+32 \ln 2\right]+C_{A} n_{f}\left[\frac{272}{9}-\frac{40}{9} \gamma_{e}-\frac{8}{3} \gamma_{e}^{2}-\frac{4}{3} \pi^{2}\right.\right. \\
& \left.-\frac{368}{9} \ln 2+\frac{16}{3} \gamma_{e} \ln 2+\frac{64}{3} \ln ^{2} 2\right]+C_{A}^{2}\left[-\frac{6200}{9}+448 \zeta_{3}-\frac{2324}{9} \gamma_{e}-\frac{124}{3} \gamma_{e}^{2}+32 \gamma_{e}^{3}+46 \pi^{2}+\frac{44}{3} \pi^{2} \gamma_{e}\right. \\
& \left.+\frac{11624}{9} \ln 2+\frac{248}{3} \gamma_{e} \ln 2-96 \gamma_{e}^{2} \ln 2-\frac{344}{3} \pi^{2} \ln 2-\frac{4192}{3} \ln ^{2} 2+96 \gamma_{e} \ln ^{2} 2+832 \ln ^{3} 2\right] \\
& +\ln \left(\mu_{f}^{2} / \mu_{r}^{2}\right) C_{A} n_{f}\left[-\frac{16}{3}+\frac{8}{3} \gamma_{e}+\frac{16}{3} \ln 2\right]+\ln \left(\mu_{f}^{2} / \mu_{r}^{2}\right) C_{A}^{2}\left[\frac{88}{3}+\frac{340}{3} \gamma_{e}-32 \gamma_{e}^{2}-16 \pi^{2}-\frac{280}{3} \ln 2\right. \\
& \left.\left.+32 \gamma_{e} \ln 2+192 \ln ^{2} 2\right]\right\}
\end{aligned}
$$

where $C_{q \bar{q}}^{(1)}=36 \pi a_{q}^{0}+\left(n_{f}-4\right)(2 / 3 \ln 2-5 / 9)$ and $C_{g g}^{(1)}=768 / 7 \pi a_{g}^{0}$ with the numerical constants $a_{q}^{0}=0.180899$ and $a_{g}^{0}=0.108068$ being reported in Table 1 of Ref. [4]. The presently unknown two-loop constants are denoted $C_{q \bar{q}}^{(2)}$ and $C_{g g}^{(2)}$.

At first and second order in $\alpha_{s}$ the Coulomb corrections have to be added to the cross section. In the limit $\rho \rightarrow 1$ (that is $\beta \rightarrow 0$ ), they read $[33,38]$ for the color-singlet final state

$$
\begin{gathered}
\hat{\sigma}_{i j, 1}^{(1), \mathrm{c}}=\hat{\sigma}_{i j, 1}^{(0)} 2 C_{F} \frac{\pi^{2}}{\beta}, \\
\hat{\sigma}_{i j, 1}^{(2), \mathrm{c}}=\hat{\sigma}_{i j, 1}^{(0)} 2 C_{F}\left\{\left(\frac{31}{9} C_{A}-16 C_{F}-\frac{10}{9} n_{f}\right.\right. \\
\left.\left.-2 \beta_{0} \ln 2-2 \beta_{0} \ln \beta\right) \frac{\pi^{2}}{\beta}+\frac{2}{3} C_{F} \frac{\pi^{4}}{\beta^{2}}\right\} .
\end{gathered}
$$

From these expressions the octet results for $\hat{\sigma}_{i j, 8}^{(1), \mathrm{c}}$ and $\hat{\sigma}_{i j, 8}^{(2), \mathrm{c}}$ are obtained by the replacement of the color factor $2 C_{F} \rightarrow$ $\left(2 C_{F}-C_{A}\right)$ consistent with results from potential nonrelativistic QCD (e.g. [38,39]).

Next we give the Mellin transforms of powers of logarithms in $\beta$ according to Eq. (7). Recall that $\rho=4 m_{t}^{2} / s$ and $\beta=\sqrt{1-4 m_{t}^{2} / s}=\sqrt{1-\rho}$. In the limit $\rho \rightarrow 1$ as needed in the fixed order expansions and accurate up to power suppressed terms in $N$, we have 


$$
\begin{aligned}
\int_{0}^{1} d \rho \rho^{N} \beta \ln ^{4} \beta= & \frac{1}{16} \ln ^{4} \tilde{N}+\left\{-\frac{1}{2}+\frac{1}{2} \ln 2\right\} \ln ^{3} \tilde{N}+\left\{-3 \ln 2+\frac{3}{16} \pi^{2}+\frac{3}{2} \ln ^{2} 2\right\} \ln ^{2} \tilde{N}+\left\{-\frac{3}{4} \pi^{2}-6 \ln ^{2} 2+\frac{3}{4} \pi^{2} \ln 2\right. \\
& \left.\left.+\frac{7}{2} \zeta_{3}+2 \ln ^{3} 2\right\} \ln \tilde{N}-7 \zeta_{3}-4 \ln ^{3} 2+\frac{7}{64} \pi^{4}+7 \zeta_{3} \ln 2+\ln ^{4} 2+\frac{3}{4} \pi^{2} \ln ^{2} 2-\frac{3}{2} \pi^{2} \ln 2\right) \\
& \times \frac{\sqrt{\pi}}{2} \frac{1}{N^{3 / 2}}(1+O(1 / N)), \\
\int_{0}^{1} d \rho \rho^{N} \beta \ln ^{3} \beta= & \left(-\frac{1}{8} \ln ^{3} \tilde{N}+\left\{-\frac{3}{4} \ln 2+\frac{3}{4}\right\} \ln ^{2} \tilde{N}+\left\{3 \ln 2-\frac{3}{2} \ln ^{2} 2-\frac{3}{16} \pi^{2}\right\} \ln \tilde{N}-\frac{7}{4} \zeta_{3}-\ln ^{3} 2+3 \ln ^{2} 2+\frac{3}{8} \pi^{2}\right. \\
& \left.-\frac{3}{8} \pi^{2} \ln 2\right) \frac{\sqrt{\pi}}{2} \frac{1}{N^{3 / 2}}(1+O(1 / N)) \\
\int_{0}^{1} d \rho \rho^{N} \beta \ln ^{2} \beta= & \left(\frac{1}{4} \ln ^{2} \tilde{N}+\{\ln 2-1\} \ln \tilde{N}+\frac{1}{8} \pi^{2}-2 \ln 2+\ln ^{2} 2\right) \frac{\sqrt{\pi}}{2} \frac{1}{N^{3 / 2}}(1+O(1 / N)), \\
\int_{0}^{1} d \rho \rho^{N} \beta \ln \beta= & \left(-\frac{1}{2} \ln \tilde{N}+1-\ln 2\right) \frac{\sqrt{\pi}}{2} \frac{1}{N^{3 / 2}}(1+O(1 / N)), \\
\int_{0}^{1} d \rho \rho^{N} \ln ^{2} \beta= & \left(\frac{1}{4} \ln { }^{2} \tilde{N}+\frac{1}{24} \pi^{2}\right) \frac{1}{N}(1+O(1 / N)), \\
\int_{0}^{1} d \rho \rho^{N} \ln \beta= & -\frac{1}{2} \ln \tilde{N} \frac{1}{N}(1+O(1 / N)),
\end{aligned}
$$

where $\tilde{N}=N \exp \left(\gamma_{e}\right)$.

Finally, we present analytical results in the $\overline{\mathrm{MS}}$ scheme for the threshold expansion of the inclusive partonic cross sections $\hat{\sigma}_{i j \rightarrow t \bar{t}}\left(s, m^{2}, \mu^{2}\right)$. The inverse powers of $\beta$ originate from the Coulomb corrections and $n_{f}$ denotes the number of effectively massless quark flavors. We set $\mu=m_{t}$ and find for the $q \bar{q}$ channel

$$
\begin{aligned}
\hat{\sigma}_{q \bar{q}}^{(1)} & =\hat{\sigma}_{q \bar{q}}^{(0)}\left\{32 C_{F} \ln ^{2} \beta+\left(96 C_{F} \ln 2-64 C_{F}-8 C_{A}\right) \ln \beta+\left(2 C_{F}-C_{A}\right) \frac{\pi^{2}}{\beta}+4 C_{q \bar{q}}^{(1)}-96 C_{F} \ln 2+72 C_{F} \ln ^{2} 2-12 C_{A} \ln 2\right\}, \\
\hat{\sigma}_{q \bar{q}}^{(2)}= & \hat{\sigma}_{q \bar{q}}^{(0)}\left\{512 C_{F}^{2} \ln ^{4} \beta+\left(\frac{256}{9} C_{F} n_{f}-2048 C_{F}^{2}+3072 C_{F}^{2} \ln 2-\frac{3712}{9} C_{A} C_{F}\right) \ln ^{3} \beta+\left(128 C_{F} C_{q \bar{q}}^{(1)}-\frac{1088}{9} C_{F} n_{f}\right.\right. \\
& +128 C_{F} n_{f} \ln 2+4096 C_{F}^{2}-256 C_{F}^{2} \pi^{2}-9216 C_{F}^{2} \ln 2+6912 C_{F}^{2} \ln ^{2} 2-\frac{32}{3} C_{A} n_{f}+\frac{10976}{9} C_{A} C_{F}-\frac{32}{3} C_{A} C_{F} \pi^{2} \\
& \left.-1856 C_{A} C_{F} \ln 2+\frac{272}{3} C_{A}^{2}\right) \ln ^{2} \beta+\left(64 C_{F}^{2}-32 C_{A} C_{F}\right) \ln 2 \beta \frac{\pi^{2}}{\beta}+\left(384 C_{F} C_{q \bar{q}}^{(1)} \ln 2-256 C_{F} C_{q \bar{q}}^{(1)}+\frac{6976}{27} C_{F} n_{f}\right. \\
& -\frac{32}{3} C_{F} n_{f} \pi^{2}-\frac{1088}{3} C_{F} n_{f} \ln 2+192 C_{F} n_{f} \ln ^{2} 2-8192 C_{F}^{2}+3584 C_{F}^{2} \zeta_{3}+512 C_{F}^{2} \pi^{2}+12288 C_{F}^{2} \ln 2-768 C_{F}^{2} \pi^{2} \ln 2 \\
& -13824 C_{F}^{2} \ln ^{2} 2+6912 C_{F}^{2} \ln 32-32 C_{A} C_{q \bar{q}}^{(1)}+\frac{272}{9} C_{A} n_{f}-32 C_{A} n_{f} \ln 2-\frac{55264}{27} C_{A} C_{F}+112 C_{A} C_{F} \zeta_{3}+144 C_{A} C_{F} \pi^{2} \\
& \left.+\frac{10976}{3} C_{A} C_{F} \ln 2-32 C_{A} C_{F} \pi^{2} \ln 2-2784 C_{A} C_{F} \ln ^{2} 2-\frac{1592}{9} C_{A}^{2}+\frac{8}{3} C_{A}^{2} \pi^{2}+272 C_{A}^{2} \ln 2\right) \ln \beta+\left(\frac{8}{3} C_{F} n_{f}+64 C_{F}^{2} \ln 2\right. \\
& \left.-\frac{4}{3} C_{A} n_{f}-\frac{92}{3} C_{A} C_{F}-32 C_{A} C_{F} \ln 2+\frac{46}{3} C_{A}^{2}\right) \ln \beta \frac{\pi^{2}}{\beta}+\frac{4}{3}\left(C_{F}-\frac{1}{2} C_{A}\right)^{2} \frac{\pi^{4}}{\beta^{2}}+\left(\frac{8}{3} C_{F} n_{f} \ln 2-\frac{20}{9} C_{F} n_{f}-32 C_{F}^{2}\right. \\
& \left.\left.+\frac{10}{9} C_{A} n_{f}-\frac{4}{3} C_{A} n_{f} \ln 2+\frac{350}{9} C_{A} C_{F}-\frac{44}{3} C_{A} C_{F} \ln 2-\frac{103}{9} C_{A}^{2}+\frac{22}{3} C_{A}^{2} \ln 2\right) \frac{\pi^{2}}{\beta}+C_{q \bar{q}}^{(2)}\right\},
\end{aligned}
$$

where $C_{q \bar{q}}^{(1)}$ has been given below Eq. (A13) and $C_{q \bar{q}}^{(2)}$ is presently unknown. For the $g g$ channel at scale $\mu=m_{t}$, we find

$$
\begin{aligned}
\hat{\sigma}_{g g}^{(1)}= & \hat{\sigma}_{g g}^{(0)}\left\{32 C_{A} \ln ^{2} \beta+\left(96 C_{A} \ln 2-72 C_{A}+16 C_{A} \frac{1}{C_{A}^{2}-2}\right) \ln \beta+\left(2 C_{F}-C_{A}+2 C_{A} \frac{1}{C_{A}^{2}-2}\right) \frac{\pi^{2}}{\beta}+4 C_{g g}^{(1)}\right. \\
& \left.-108 C_{A} \ln 2+72 C_{A} \ln ^{2} 2+24 C_{A} \ln 2 \frac{1}{C_{A}^{2}-2}\right\},
\end{aligned}
$$




$$
\begin{aligned}
\hat{\sigma}_{g g}^{(2)}= & \hat{\sigma}_{g g}^{(0)}\left\{512 C_{A}^{2} \ln ^{4} \beta+\left(512+\frac{256}{9} C_{A} n_{f}-\frac{22144}{9} C_{A}^{2}+3072 C_{A}^{2} \ln 2+1024 \frac{1}{C_{A}^{2}-2}\right) \ln ^{3} \beta+\left(2304 \ln 2-\frac{3616}{3}\right.\right. \\
& +128 C_{A} C_{g g}^{(1)}-\frac{1184}{9} C_{A} n_{f}+128 C_{A} n_{f} \ln 2+\frac{48656}{9} C_{A}^{2}-\frac{800}{3} C_{A}^{2} \pi^{2}-11072 C_{A}^{2} \ln 2+6912 C_{A}^{2} \ln ^{2} 2 \\
& \left.+\left[4608 \ln 2-\frac{7232}{3}+\frac{64}{3} C_{A} n_{f}\right] \frac{1}{C_{A}^{2}-2}\right) \ln ^{2} \beta+\left(64+64 C_{A} C_{F}-32 C_{A}^{2}+128 \frac{1}{C_{A}^{2}-2}\right) \ln ^{2} \beta \frac{\pi^{2}}{\beta} \\
& +\left(\frac{12400}{9}-\frac{400}{3} \pi^{2}-3616 \ln 2+3456 \ln ^{2} 2-288 C_{A} C_{g g}^{(1)}+384 C_{A} C_{g g}^{(1)} \ln 2+\frac{7792}{27} C_{A} n_{f}-\frac{32}{3} C_{A} n_{f} \pi^{2}\right. \\
& -\frac{1184}{3} C_{A} n_{f} \ln 2+192 C_{A} n_{f} \ln ^{2} 2-\frac{281224}{27} C_{A}^{2}+3696 C_{A}^{2} \zeta_{3}+\frac{1976}{3} C_{A}^{2} \pi^{2}+\frac{48656}{3} C_{A}^{2} \ln 2-800 C_{A}^{2} \pi^{2} \ln 2 \\
& -16608 C_{A}^{2} \ln 2+6912 C_{A}^{2} \ln ^{3} 2+\left[\frac{24800}{9}-\frac{800}{3} \pi^{2}-7232 \ln 2+6912 \ln ^{2} 2+64 C_{A} C_{g g}^{(1)}-\frac{544}{9} C_{A} n_{f}\right. \\
& \left.\left.+64 C_{A} n_{f} \ln 2\right] \frac{1}{C_{A}^{2}-2}\right) \ln \beta+\left(64 \ln 2-\frac{44}{3}+\frac{8}{3} C_{F} n_{f}-\frac{4}{3} C_{A} n_{f}-\frac{92}{3} C_{A} C_{F}+64 C_{A} C_{F} \ln 2+\frac{46}{3} C_{A}^{2}\right. \\
& \left.-32 C_{A}^{2} \ln 2+\left[128 \ln 2-\frac{136}{3}+\frac{8}{3} C_{A} n_{f}\right] \frac{1}{C_{A}^{2}-2}\right) \ln \beta \frac{\pi^{2}}{\beta}+\left[\frac{4}{3}\left(C_{F}-\frac{1}{2} C_{A}\right)^{2}+\frac{2}{3}\right] \frac{\pi^{4}}{\beta^{2}}+\left(-\frac{82}{9}-\frac{44}{3} \ln 2\right. \\
& -\frac{20}{9} C_{F} n_{f}+\frac{8}{3} C_{F} n_{f} \ln 2-32 C_{F}^{2}+\frac{10}{9} C_{A} n_{f}-\frac{4}{3} C_{A} n_{f} \ln 2+\frac{350}{9} C_{A} C_{F}-\frac{44}{3} C_{A} C_{F} \ln 2-\frac{103}{9} C_{A}^{2} \\
& \left.\left.+\frac{22}{3} C_{A}^{2} \ln 2+\left[\frac{124}{9}-\frac{88}{3} \ln 2-\frac{20}{9} C_{A} n_{f}+\frac{8}{3} C_{A} n_{f} \ln 2\right] \frac{1}{C_{A}^{2}-2}\right) \frac{\pi^{2}}{\beta}+C_{g g}^{(2)}\right\},
\end{aligned}
$$

\begin{tabular}{|c|c|c|c|c|c|c|c|}
\hline PDF set & $\sigma_{\mathrm{NLO}}$ & $\sigma_{\text {res }}$ & $\sigma_{\mathrm{NNLO}(\text { approx) }}$ & PDF set & $\sigma_{\mathrm{NLO}}$ & $\sigma_{\text {res }}$ & $\sigma_{\mathrm{NNLO}(\text { approx) }}$ \\
\hline 0 & $925_{+112}^{-108}$ & $932_{+105}^{-94}$ & $969_{-39}^{-13}$ & & & & \\
\hline 1 & $920_{+111}^{-108}$ & $\begin{array}{l}927^{-94} \\
+105\end{array}$ & $964_{-39}^{-12}$ & 2 & $929_{+112}^{-109}$ & $\begin{array}{l}937_{+106}^{-95} \\
\text { - }\end{array}$ & $974_{-39}^{-13}$ \\
\hline 3 & $924_{+111}^{-108}$ & $\begin{array}{l}931_{+105}^{-94} \\
\text { S }\end{array}$ & $968_{-39}^{-12}$ & 4 & $925_{+112}^{-109}$ & $\begin{array}{l}933^{-95}+106 \\
\end{array}$ & $970_{-39}^{-13}$ \\
\hline 5 & $928_{+112}^{-111}$ & $\begin{array}{l}935_{+106}^{-95} \\
9\end{array}$ & $972_{-39}^{-13}$ & 6 & $922_{+111}^{-108}$ & $929_{+105}^{-94}$ & $966_{-39}^{-12}$ \\
\hline 7 & $925_{+112}^{-108}$ & $932_{+105}^{-94}$ & $969_{-39}^{-13}$ & 8 & $924_{+112}^{-111}$ & $931_{+105}^{-94}$ & $969_{-39}^{-13}$ \\
\hline 9 & $926_{+112}^{-109}$ & $933_{+105}^{-94}$ & $970_{-39}^{-13}$ & 10 & $923_{+112}^{-108}$ & $931_{+105}^{-94}$ & $968_{-39}^{-12}$ \\
\hline 11 & $927_{+112}^{-112}$ & $934_{+106}^{-105}$ & $972_{-39}^{-12}$ & 12 & $922_{+112}^{-108}$ & $929_{+105}^{-94}$ & $966_{-39}^{-13}$ \\
\hline 13 & $926_{+112}^{-108}$ & $934_{+105}^{-94}$ & $971_{-39}^{-13}$ & 14 & $923_{+112}^{-112}$ & $\begin{array}{l}930_{+105}^{+105} \\
9\end{array}$ & $967_{-39}^{-13}$ \\
\hline 15 & $929_{+112}^{-112}$ & $936_{+106}^{-105}$ & $973_{-39}^{-13}$ & 16 & $920_{+111}^{-108}$ & $927_{+105}^{-105}$ & $965_{-39}^{-12}$ \\
\hline 17 & $916_{+111}^{-108}$ & $924_{+104}^{-94}$ & $961_{-39}^{-13}$ & 18 & $932_{+112}^{-109}$ & $939_{+106}^{-94}$ & $976_{-40}^{-12}$ \\
\hline 19 & $922_{+111}^{-108}$ & $929_{+105}^{-94}$ & $966_{-39}^{-12}$ & 20 & $926_{+112}^{-109}$ & $\begin{array}{l}933^{-95}+106 \\
\end{array}$ & $\begin{array}{l}971_{-39}^{-13} \\
\end{array}$ \\
\hline 21 & $925_{+112}^{-108}$ & $932_{+105}^{-94}$ & $970_{-39}^{-13}$ & 22 & $924_{+112}^{-108}$ & $931_{+105}^{+106}$ & $968_{-39}^{-13}$ \\
\hline 23 & $924_{+112}^{-109}$ & $931_{+105}^{-94}$ & $969_{-39}^{-13}$ & 24 & $925_{+112}^{-108}$ & $933_{+105}^{-94}$ & $970_{-39}^{-12}$ \\
\hline 25 & $924_{+112}^{-108}$ & $931_{+105}^{-94}$ & $969_{-39}^{-12}$ & 26 & $925_{+112}^{-108}$ & $932_{+105}^{-105}$ & $969_{-39}^{-13}$ \\
\hline 27 & $926_{+112}^{-108}$ & $933_{+105}^{-94}$ & $970_{-39}^{-13}$ & 28 & $925_{+112}^{-109}$ & $932_{+105}^{-95}$ & $970_{-39}^{-13}$ \\
\hline 29 & $924_{+111}^{-108}$ & $931_{+105}^{-94}$ & $968_{-39}^{-12}$ & 30 & $924_{+112}^{-108}$ & $931_{+105}^{-94}$ & $969_{-39}^{-12}$ \\
\hline
\end{tabular}

and $C_{g g}^{(1)}$ has again been given below Eq. (A13) while $C_{g g}^{(2)}$ is the yet uncalculated two-loop constant.

\section{APPENDIX B: DETAILED RESULTS FOR SPECIFIC PDFS}

In Tables X, XI, XII, and XIII, we present detailed theory predictions for the total cross section at Tevatron and LHC for the individual PDFs and their complete set of eigenvalues.

TABLE X. The total cross section for $\mu=m_{t}$ at LHC for $m_{t}=171 \mathrm{GeV}$ and the full set of predictions from the MRST-2006 NNLO PDF set [25]. All rates are in pb. We denote by $\sigma_{\mathrm{NLO}}$ [4,5] the NLO QCD prediction, by $\sigma_{\text {res }}$ the result of NLL threshold resummation [8], and by $\sigma_{\mathrm{NNLO} \text { (approx) }}$ the NNLO QCD prediction based on soft-gluon approximation and exact two-loop scale dependence [9]. The upper and lower indices denote the shifts towards $\mu=2 m_{t}$ and $\mu=m_{t} / 2$. 
TABLE XI. The total cross section for $\mu=m_{t}$ at LHC for $m_{t}=171 \mathrm{GeV}$ and the full set of predictions from the CTEQ6.5 PDF set [24]. All rates are in pb. We denote by $\sigma_{\mathrm{NLO}}[4,5]$ the NLO QCD prediction, by $\sigma_{\text {res }}$ the result of NLL threshold resummation [8], and by $\sigma_{\mathrm{NNLO}(\text { approx) }}$ the NNLO QCD prediction based on soft-gluon approximation and exact two-loop scale dependence [9]. The upper and lower indices denote the shifts towards $\mu=2 m_{t}$ and $\mu=m_{t} / 2$.

\begin{tabular}{|c|c|c|c|c|c|c|c|}
\hline PDF set & $\sigma_{\mathrm{NLO}}$ & $\sigma_{\text {res }}$ & $\sigma_{\mathrm{NNLO}(\text { approx) }}$ & PDF set & $\sigma_{\mathrm{NLO}}$ & $\sigma_{\text {res }}$ & $\sigma_{\mathrm{NNLO}(\text { approx) }}$ \\
\hline 0 & $875_{+102}^{-101}$ & $882_{+95}^{-87}$ & $918_{-39}^{-9}$ & & & & \\
\hline 1 & $877_{+102}^{-101}$ & $884_{+96}^{-87}$ & $920_{-39}^{-9}$ & 2 & $873_{+102}^{-101}$ & $880_{+95}^{-87}$ & $916_{-39}^{-9}$ \\
\hline 3 & $878_{+102}^{-101}$ & $885_{+96}^{-88}$ & $921_{-39}^{-9}$ & 4 & $872_{+101}^{-101}$ & $879_{+95}^{-87}$ & $915_{-39}^{-9}$ \\
\hline 5 & $877_{+102}^{-101}$ & $884_{+96}^{-88}$ & $921_{-39}^{-9}$ & 6 & $872_{+101}^{-100}$ & $879_{+95}^{-87}$ & $915_{-39}^{-9}$ \\
\hline 7 & $883_{+103}^{-103}$ & $890_{+97}^{-89}$ & $926_{-40}^{-10}$ & 8 & $867_{+100}^{-99}$ & $874_{+94}^{-86}$ & $909_{-39}^{-9}$ \\
\hline 9 & $877_{+102}^{-101}$ & $884_{+96}^{-87}$ & $920_{-39}^{-9}$ & 10 & $872_{+102}^{-101}$ & $879_{+95}^{-87}$ & $915_{-39}^{-9}$ \\
\hline 11 & $899_{+105}^{-103}$ & $906_{+98}^{-89}$ & $943_{-40}^{-9.6}$ & 12 & $\begin{array}{l}852_{+99}^{-99} \\
\end{array}$ & $858_{+93}^{-85}$ & $894_{-38}^{-9}$ \\
\hline 13 & $876_{+102}^{-101}$ & $883_{+96}^{-88}$ & $919_{-39}^{-9}$ & 14 & $874_{+101}^{-101}$ & $881_{+95}^{-87}$ & $917_{-39}^{-9}$ \\
\hline 15 & $869_{+102}^{-101}$ & $876_{+95}^{-87}$ & $912_{-39}^{-9}$ & 16 & $880_{+102}^{-101}$ & $\begin{array}{l}887_{+96}^{-87} \\
\end{array}$ & $923_{-39}^{-9}$ \\
\hline 17 & $880_{+103}^{-102}$ & $888_{+96}^{-88}$ & $\begin{array}{l}924_{-40}^{-9.5} \\
\text {. }\end{array}$ & 18 & $869_{+101}^{-100}$ & $876_{+95}^{-86}$ & $912_{-39}^{-9}$ \\
\hline 19 & $878_{+102}^{-101}$ & $885_{+96}^{-88}$ & $921_{-39}^{-9}$ & 20 & $871_{+101}^{-101}$ & $878_{+95}^{-87}$ & $914_{-39}^{-9}$ \\
\hline 21 & $870_{+101}^{-101}$ & $877_{+95}^{-87}$ & $913_{-39}^{-9}$ & 22 & $880_{+102}^{-101}$ & $\begin{array}{l}887_{+96}^{-87} \\
\end{array}$ & $923_{-40}^{-9}$ \\
\hline 23 & $867_{+101}^{-100}$ & $874_{+95}^{-87}$ & $910_{-39}^{-9}$ & 24 & $885_{+103}^{-102}$ & $\begin{array}{l}892_{+96}^{-88} \\
\end{array}$ & $928_{-40}^{-9}$ \\
\hline 25 & $875_{+101}^{-99}$ & $882_{+95}^{-86}$ & $917_{-39}^{-8}$ & 26 & $875_{+102}^{-102}$ & $882_{+96}^{-88}$ & $919_{-39}^{-9.7}$ \\
\hline 27 & $874_{+102}^{-101}$ & $881_{+95}^{-87}$ & $917_{-39}^{-9}$ & 28 & $875_{+102}^{-101}$ & $882_{+96}^{-87}$ & $918_{-39}^{-9}$ \\
\hline 29 & $876_{+102}^{-101}$ & $883_{+96}^{-88}$ & $920_{-39}^{-9}$ & 30 & $873_{+102}^{-101}$ & $880_{+95}^{-87}$ & $916_{-39}^{-9}$ \\
\hline 31 & $868_{+101}^{-99.9}$ & $875_{+95}^{-86}$ & $911_{-39}^{-9}$ & 32 & $878_{+102}^{-102}$ & $886_{+96}^{-88}$ & $922_{-39}^{-9.5}$ \\
\hline 33 & $\begin{array}{l}875_{+102}^{-102} \\
\end{array}$ & $882_{+96}^{-88}$ & $918_{-39}^{-9.6}$ & 34 & $874_{+101}^{-100}$ & $881_{+95}^{-86}$ & $917_{-39}^{-9}$ \\
\hline 35 & $873_{+102}^{-101}$ & $880_{+95}^{-87}$ & $916_{-39}^{-9}$ & 36 & $874_{+102}^{-101}$ & $881_{+95}^{-87}$ & $917_{-39}^{-9}$ \\
\hline 37 & $875_{+102}^{-101}$ & $882_{+95}^{-87}$ & $918_{-39}^{-9}$ & 38 & $872_{+102}^{-101}$ & $879_{+95}^{-87}$ & $916_{-39}^{-9}$ \\
\hline 39 & $874_{+102}^{-101}$ & $881_{+95}^{-87}$ & $917_{-39}^{-9}$ & 40 & $875_{+102}^{-101}$ & $882_{+96}^{-87}$ & $918_{-39}^{-9}$ \\
\hline
\end{tabular}

TABLE XII. The total cross section for $\mu=m_{t}$ at Tevatron for $m_{t}=171 \mathrm{GeV}$ and the full set of predictions from the CTEQ6.5 PDF set [24]. All rates are in pb. We denote by $\sigma_{\mathrm{NLO}}$ [4,5] the NLO QCD prediction, by $\sigma_{\text {res }}$ the result of NLL threshold resummation [8], and by $\sigma_{\mathrm{NNLO} \text { (approx) }}$ the NNLO QCD prediction based on soft-gluon approximation and exact two-loop scale dependence [9]. The upper and lower indices denote the shifts towards $\mu=2 m_{t}$ and $\mu=m_{t} / 2$.

\begin{tabular}{|c|c|c|c|c|c|c|c|}
\hline PDF set & $\sigma_{\mathrm{NLO}}$ & $\sigma_{\text {res }}$ & $\sigma_{\mathrm{NNLO}(\text { approx) }}$ & PDF set & $\sigma_{\mathrm{NLO}}$ & $\sigma_{\text {res }}$ & $\sigma_{\mathrm{NNLO}(\text { approx) }}$ \\
\hline 0 & $7.35_{+0.38}^{-0.80}$ & $7.53_{+0.25}^{-0.66}$ & $7.94_{+0.07}^{-0.28}$ & & & & \\
\hline 1 & $7.48_{+0.38}^{-0.81}$ & $\begin{array}{l}7.67_{+0.25}^{-0.67} \\
\end{array}$ & $8.08_{+0.07}^{-0.29}$ & 2 & $7.23_{+0.37}^{-0.79}$ & $7.41_{+0.25}^{-0.65}$ & $7.81_{+0.07}^{-0.28}$ \\
\hline 3 & $7.35_{+0.38}^{-0.80}$ & $7.54_{+0.25}^{-0.66}$ & $7.94_{+0.07}^{-0.28}$ & 4 & $7.35_{+0.38}^{-0.80}$ & $7.53_{+0.25}^{-0.66}$ & $7.94_{+0.07}^{-0.28}$ \\
\hline 5 & $7.37_{+0.38}^{-0.80}$ & $7.55_{+0.65}^{+0.25}$ & $7.96_{+0.07}^{-0.07}$ & 6 & $7.33_{+0.38}^{-0.38}$ & $7.52_{+0.25}^{-0.66}$ & $7.92_{+0.07}^{+0.07}$ \\
\hline 7 & $\begin{array}{r}7.22_{+0.39}^{-0.78} \\
\end{array}$ & $7.40_{+0.25}^{-0.65}$ & $7.81_{+0.07}^{-0.28}$ & 8 & $7.49_{+0.38}^{-0.81}$ & $7.67_{+0.25}^{-0.67}$ & $8.09_{+0.07}^{-0.28}$ \\
\hline 9 & $7.38_{+0.38}^{-0.81}$ & $7.56_{+0.26}^{-0.66}$ & $\begin{array}{r}7.97_{+0.07}^{-0.28}\end{array}$ & 10 & $7.32_{+0.37}^{-0.80}$ & $7.51_{+0.25}^{-0.66}$ & $7.91_{+0.07}^{-0.028}$ \\
\hline 11 & $7.44_{+0.41}^{-0.80}$ & $7.62_{+0.28}^{-0.60}$ & $8.04_{+0.06}^{-0.29}$ & 12 & $7.29_{+0.78}^{-0.31}$ & $7.47_{+0.23}^{-0.63}$ & $7.87_{+0.08}^{-0.27}$ \\
\hline 13 & $7.34_{+0.38}^{-0.80}$ & $\begin{array}{l}7.52_{+0.25}^{-0.66} \\
\text {. }\end{array}$ & $7.93_{+0.07}^{-0.28}$ & 14 & $7.36_{+0.38}^{-0.80}$ & $7.54_{+0.25}^{-0.66}$ & $7.95_{+0.07}^{-0.28}$ \\
\hline 15 & $\begin{array}{r}7.27_{+0.79}^{-0.79} \\
\end{array}$ & $7.45_{+0.24}^{-0.65}$ & $7.85_{+0.07}^{-0.01}$ & 16 & $7.44_{+0.39}^{-0.38}$ & $7.63_{+0.26}^{+0.67}$ & $8.04_{+0.07}^{-0.29}$ \\
\hline 17 & $7.29_{+0.38}^{-0.80}$ & $7.47^{-0.66}$ & $7.87_{+0.07}^{-0.28}$ & 18 & $7.40_{+0.38}^{-0.80}$ & $7.59^{-0.66}$ & $8.00_{+0.07}^{-0.28}$ \\
\hline 19 & $7.28_{+0.38}^{-0.80}$ & $7.46_{+0.26}^{-0.66}$ & $7.87_{+0.07}^{-0.28}$ & 20 & $\begin{array}{l}7.43_{+0.37}^{-0.80} \\
\end{array}$ & $7.61_{+0.25}^{-0.66}$ & $8.02_{+0.07}^{-0.28}$ \\
\hline 21 & $\begin{array}{r}7.29_{+0.39}^{-0.79}\end{array}$ & $\begin{array}{l}7.47_{+0.24}^{-0.65}\end{array}$ & $\begin{array}{l}7.87_{+0.07}^{-0.28}\end{array}$ & 22 & $7.44_{+0.40}^{-0.82}$ & $7.62_{+0.27}^{-0.68}$ & $8.04_{+0.06}^{-0.29}$ \\
\hline 23 & $\begin{array}{l}7.25_{+0.36}^{-0.78} \\
\end{array}$ & $7.43_{+0.23}^{-0.64}$ & $7.82_{+0.08}^{-0.27}$ & 24 & $7.50_{+0.41}^{-0.83}$ & $7.69_{+0.28}^{-0.69}$ & $8.11_{+0.06}^{-0.29}$ \\
\hline 25 & $\begin{array}{l}7.71_{+0.45}^{-0.808} \\
\end{array}$ & $7.90_{+0.32}^{-0.72}$ & $8.34_{+0.05}^{-0.30}$ & 26 & $7.19_{+0.34}^{-0.41}$ & $\begin{array}{l}7.37^{-0.63} \\
+0.22\end{array}$ & $7.76_{+0.08}^{-0.27}$ \\
\hline 27 & $\begin{array}{l}7.41_{+0.39}^{-0.81}\end{array}$ & $\begin{array}{l}7.59_{+0.26}^{-0.67} \\
\end{array}$ & $8.01_{+0.07}^{-0.29}$ & 28 & $7.29_{+0.37}^{-0.79}$ & $7.48_{+0.24}^{-0.65}$ & $7.88_{+0.07}^{-0.28}$ \\
\hline 29 & $7.35_{+0.38}^{-0.80}$ & $7.54_{+0.25}^{-0.66}$ & $7.94_{+0.07}^{-0.28}$ & 30 & $7.34_{+0.38}^{-0.80}$ & $7.52_{+0.25}^{-0.66}$ & $7.93_{+0.07}^{-0.28}$ \\
\hline 31 & $\begin{array}{l}7.42_{+0.39}^{-0.81}\end{array}$ & $7.60_{+0.26}^{-0.67}$ & $8.01_{+0.07}^{-0.29}$ & 32 & $7.27_{+0.37}^{-0.78}$ & $7.45_{+0.24}^{-0.65}$ & $7.85_{+0.07}^{-0.02}$ \\
\hline 33 & $7.27_{+0.39}^{-0.39}$ & $7.46_{+0.24}^{+0.20}$ & $7.86_{+0.08}^{-0.01}$ & 34 & $7.44_{+0.40}^{-0.31}$ & $7.63_{+0.27}^{-0.64}$ & $8.04_{+0.06}^{-0.29}$ \\
\hline 35 & $\begin{array}{r}7.39_{+0.31}^{-0.80} \\
\end{array}$ & $7.58_{+0.26}^{-0.67}$ & $7.99_{+0.07}^{-0.28}$ & 36 & $7.32_{+0.37}^{-0.80}$ & $7.50_{+0.25}^{-0.66}$ & $7.91_{+0.07}^{-0.28}$ \\
\hline 37 & $\begin{array}{l}7.37_{+0.38}^{-0.81} \\
\end{array}$ & $7.55_{+0.26}^{-0.67}$ & $7.96_{+0.07}^{-0.28}$ & 38 & $\begin{array}{l}7.31_{+0.37}^{-0.79}\end{array}$ & $7.49_{+0.25}^{-0.65}$ & $7.90_{+0.07}^{-0.28}$ \\
\hline 39 & $7.33_{+0.38}^{-0.80}$ & $7.52_{+0.25}^{-0.66}$ & $7.92_{+0.07}^{-0.28}$ & 40 & $7.35_{+0.38}^{-0.80}$ & $7.53_{+0.25}^{-0.66}$ & $7.94_{+0.07}^{-0.28}$ \\
\hline
\end{tabular}


TABLE XIII. The total cross section for $\mu=m_{t}$ at Tevatron for $m_{t}=171 \mathrm{GeV}$ and the full set of predictions from the MRST-2006 NNLO PDF set [25]. All rates are in pb. We denote by $\sigma_{\mathrm{NLO}}[4,5]$ the NLO QCD prediction, by $\sigma_{\text {res }}$ the result of NLL threshold resummation [8], and by $\sigma_{\mathrm{NNLO} \text { (approx) }}$ the NNLO QCD prediction based on soft-gluon approximation and exact two-loop scale dependence [9]. The upper and lower indices denote the shifts towards $\mu=2 m_{t}$ and $\mu=m_{t} / 2$.

\begin{tabular}{|c|c|c|c|c|c|c|c|}
\hline$\underline{\text { PDF set }}$ & $\sigma_{\mathrm{NLO}}$ & $\sigma_{\text {res }}$ & $\sigma_{\mathrm{NNLO}(\text { approx) }}$ & PDF set & $\sigma_{\mathrm{NLO}}$ & $\sigma_{\text {res }}$ & $\sigma_{\mathrm{NNLO}(\text { approx) }}$ \\
\hline 0 & $7.60_{+0.47}^{-0.90}$ & $7.79_{+0.35}^{-0.75}$ & $8.24_{+0.08}^{-0.34}$ & & & & \\
\hline 1 & $7.59_{+0.47}^{-0.89}$ & $\begin{array}{l}7.78_{+0.34}^{-0.75} \\
\text {. }\end{array}$ & $\begin{array}{l}8.23_{+0.08}^{-0.34} \\
\end{array}$ & 2 & $7.62_{+0.48}^{-0.90}$ & $7.80_{+0.35}^{-0.75}$ & $8.26_{+0.08}^{-0.34}$ \\
\hline 3 & $7.63_{+0.48}^{-0.90}$ & $\begin{array}{l}7.81_{+0.35}^{-0.75} \\
\text {. }\end{array}$ & $\begin{array}{l}8.27_{+0.08}^{-0.34} \\
\end{array}$ & 4 & $7.58_{+0.47}^{-0.89}$ & $\begin{array}{l}7.77_{+0.34}^{-0.75} \\
\end{array}$ & $\begin{array}{l}8.22_{+0.08}^{-0.34} \\
\text { - }\end{array}$ \\
\hline 5 & $\begin{array}{r}7.55_{+0.47}^{-0.89} \\
\end{array}$ & $\begin{array}{l}7.73_{+0.34}^{-0.75} \\
\end{array}$ & $8.18_{+0.08}^{-0.34}$ & 6 & $7.66_{+0.48}^{-0.90}$ & $7.85_{+0.35}^{-0.75}$ & $8.30_{+0.08}^{-0.34}$ \\
\hline 7 & $7.58_{+0.47}^{-0.89}$ & $\begin{array}{l}7.76_{+0.34}^{-0.75} \\
\text {. }\end{array}$ & $\begin{array}{l}8.21_{+0.08}^{-0.34} \\
\end{array}$ & 8 & $7.63_{+0.47}^{-0.90}$ & $7.82_{+0.35}^{-0.75}$ & $\begin{array}{l}8.27_{+0.34}^{-0.08} \\
\end{array}$ \\
\hline 9 & $7.60_{+0.47}^{-0.90}$ & $\begin{array}{l}7.79_{+0.34}^{-0.75} \\
\text { + }\end{array}$ & $8.24_{+0.08}^{-0.34}$ & 10 & $7.60_{+0.48}^{-0.90}$ & $7.79_{+0.35}^{-0.75}$ & $8.24_{+0.08}^{-0.34}$ \\
\hline 11 & $7.63_{+0.48}^{-0.90}$ & $7.82_{+0.35}^{-0.76}$ & $\begin{array}{l}8.27_{+0.08}^{-0.34} \\
\end{array}$ & 12 & $7.58_{+0.47}^{-0.89}$ & $7.76_{+0.34}^{-0.74}$ & $\begin{array}{l}8.21_{+0.09}^{-0.34} \\
0\end{array}$ \\
\hline 13 & $7.62_{+0.48}^{-0.90}$ & $7.81_{+0.35}^{-0.75}$ & $8.26_{+0.08}^{-0.34}$ & 14 & $7.60_{+0.47}^{-0.89}$ & $7.78_{+0.34}^{-0.75}$ & $8.23_{+0.08}^{-0.34}$ \\
\hline 15 & $7.65_{+0.48}^{-0.90}$ & $7.84_{+0.35}^{-0.76}$ & $\begin{array}{l}8.29_{+0.08}^{-0.34} \\
\end{array}$ & 16 & $\begin{array}{r}7.57_{+0.47}^{-0.89} \\
\end{array}$ & $7.75_{+0.34}^{-0.74}$ & $8.20_{+0.08}^{-0.34}$ \\
\hline 17 & $7.50_{+0.45}^{-0.87}$ & $\begin{array}{l}7.68_{+0.32}^{-0.73} \\
\text {. }\end{array}$ & $\begin{array}{l}8.12_{+0.09}^{-0.33} \\
\end{array}$ & 18 & $7.74_{+0.50}^{-0.93}$ & $\begin{array}{l}7.93_{+0.37}^{-0.78} \\
\text { (1) }\end{array}$ & $8.40_{+0.08}^{-0.35}$ \\
\hline 19 & $7.70_{+0.49}^{-0.91}$ & $\begin{array}{l}7.88_{+0.36}^{-0.77} \\
\text {. }\end{array}$ & $8.34_{+0.08}^{-0.35}$ & 20 & $\begin{array}{l}7.52_{+0.46}^{-0.88} \\
\text {. }\end{array}$ & $7.71_{+0.33}^{-0.74}$ & $8.15_{+0.09}^{-0.33}$ \\
\hline 21 & $7.71_{+0.48}^{-0.91}$ & $7.90_{+0.35}^{-0.76}$ & $\begin{array}{l}8.36_{+0.08}^{-0.34} \\
\text {. }\end{array}$ & 22 & $\begin{array}{l}7.52_{+0.47}^{-0.88} \\
\text {. }\end{array}$ & $7.70_{+0.34}^{-0.74}$ & $8.15_{+0.08}^{-0.33}$ \\
\hline 23 & $\begin{array}{l}7.62_{+0.47}^{-0.89} \\
\text { (1) }\end{array}$ & $\begin{array}{l}7.81_{+0.34}^{-0.75} \\
\text {. }\end{array}$ & $8.26_{+0.09}^{-0.34}$ & 24 & $7.63_{+0.48}^{-0.90}$ & $7.81_{+0.35}^{-0.75}$ & $\begin{array}{l}8.27_{+0.08}^{-0.34} \\
\end{array}$ \\
\hline 25 & $7.65_{+0.48}^{-0.90}$ & $7.84_{+0.35}^{-0.75}$ & $\begin{array}{l}8.29_{+0.08}^{-0.34} \\
\end{array}$ & 26 & $7.62_{+0.47}^{-0.90}$ & $7.81_{+0.34}^{-0.75}$ & $8.26_{+0.08}^{-0.03}$ \\
\hline 27 & $\begin{array}{l}7.60_{+0.48}^{-0.90} \\
\end{array}$ & $\begin{array}{l}7.79_{+0.35}^{-0.75} \\
\text { - }\end{array}$ & $8.24_{+0.08}^{-0.34}$ & 28 & $\begin{array}{r}7.57_{+0.49}^{-0.89} \\
\end{array}$ & $\begin{array}{l}7.75_{+0.34}^{-0.74} \\
\end{array}$ & $\begin{array}{l}8.20_{+0.09}^{-0.34} \\
\end{array}$ \\
\hline 29 & $7.63_{+0.48}^{-0.90}$ & $\begin{array}{l}7.82_{+0.35}^{-0.75} \\
\end{array}$ & $\begin{array}{c}8.27_{+0.08}^{-0.34} \\
\end{array}$ & 30 & $7.64_{+0.47}^{-0.90}$ & $\begin{array}{r}7.83_{+0.34}^{-0.75} \\
\end{array}$ & $\begin{array}{c}8.28_{+0.08}^{-0.34} \\
2\end{array}$ \\
\hline
\end{tabular}

[1] ATLAS, Report No. CERN-LHCC-1999-015, ATLASTDR-015, 1999 (available at http://cdsweb.cern.ch/).

[2] CMS, Report No. CERN-LHCC-2006-021, CMS-TDR008-2, 2006 (available at http://cdsweb.cern.ch/).

[3] A. Quadt, Eur. Phys. J. C 48, 835 (2006).

[4] P. Nason, S. Dawson, and R. K. Ellis, Nucl. Phys. B303, 607 (1988).

[5] W. Beenakker et al., Phys. Rev. D 40, 54 (1989).

[6] W. Bernreuther et al., Nucl. Phys. B690, 81 (2004).

[7] N. Kidonakis and G. Sterman, Nucl. Phys. B505, 321 (1997).

[8] R. Bonciani et al., Nucl. Phys. B529, 424 (1998).

[9] N. Kidonakis et al., Phys. Rev. D 64, 114001 (2001).

[10] K. Hagiwara, Y. Sumino, and H. Yokoya, arXiv:0804.1014.

[11] P. M. Nadolsky et al., arXiv:0802.0007.

[12] Tevatron Electroweak Working Group (TEV EWWG) for CDF and D0, arXiv:0803.1683.

[13] N. Kidonakis and R. Vogt, Phys. Rev. D 68, 114014 (2003).

[14] M. Cacciari et al., J. High Energy Phys. 04 (2004) 068.

[15] S. Dittmaier, P. Uwer, and S. Weinzierl, Phys. Rev. Lett. 98, 262002 (2007).

[16] M. Czakon, A. Mitov, and S. Moch, Phys. Lett. B 651, 147 (2007).

[17] M. Czakon, A. Mitov, and S. Moch, Nucl. Phys. B798, 210 (2008).

[18] J. G. Körner, Z. Merebashvili, and M. Rogal, Phys. Rev. D 77, 094011 (2008)

[19] M. Czakon, arXiv:0803.1400.

[20] R. Frederix and F. Maltoni, arXiv:0712.2355.

[21] J. Pumplin et al., J. High Energy Phys. 07 (2002) 012.

[22] H. Contopanagos, E. Laenen, and G. Sterman, Nucl. Phys.
B484, 303 (1997).

[23] S. Catani et al., Nucl. Phys. B478, 273 (1996).

[24] W. K. Tung et al., J. High Energy Phys. 02 (2007) 053.

[25] A. D. Martin et al., Phys. Lett. B 652, 292 (2007).

[26] A. Vogt, Phys. Lett. B 497, 228 (2001).

[27] S. Catani et al., J. High Energy Phys. 07 (2003) 028.

[28] S. Moch, J. A. M. Vermaseren, and A. Vogt, Nucl. Phys. B726, 317 (2005).

[29] S. Moch, J. A. M. Vermaseren, and A. Vogt, Nucl. Phys. B688, 101 (2004).

[30] A. Vogt, S. Moch, and J. A. M. Vermaseren, Nucl. Phys. B691, 129 (2004).

[31] S. Moch and A. Vogt, Phys. Lett. B 631, 48 (2005).

[32] E. Laenen and L. Magnea, Phys. Lett. B 632, 270 (2006).

[33] W. Bernreuther et al., Nucl. Phys. B706, 245 (2005).

[34] A. Mitov and S. Moch, J. High Energy Phys. 05 (2007) 001.

[35] S. Mert Aybat, L. J. Dixon, and G. Sterman, Phys. Rev. Lett. 97, 072001 (2006).

[36] S. Mert Aybat, L. J. Dixon, and G. Sterman, Phys. Rev. D 74, 074004 (2006).

[37] J. Kodaira and L. Trentadue, Phys. Lett. 112B, 66 (1982).

[38] A. Czarnecki and K. Melnikov, Phys. Rev. Lett. 80, 2531 (1998).

[39] A. Pineda and A. Signer, Nucl. Phys. B762, 67 (2007).

[40] S. Alekhin, JETP Lett. 82, 628 (2005).

[41] CDF, Report No. CDF-note-8148, 2006 (available at http://www-cdf.fnal.gov).

[42] M. Dittmar, F. Pauss, and D. Zürcher, Phys. Rev. D 56, 7284 (1997)

[43] M. Dittmar et al., arXiv:hep-ph/0511119.

[44] M. Cacciari et al., arXiv:0804.2800. 